\title{
The Current State of Pharmacological Treatments for Cannabis Use Disorder and Withdrawal
}

\author{
Christina A Brezing ${ }^{\star, 1,2}$ and Frances R Levin ${ }^{1,2}$ \\ ${ }^{1}$ Division of Substance Use Disorders, New York State Psychiatric Institute, New York, NY, USA; ${ }^{2}$ Department of Psychiatry, \\ College of Physicians and Surgeons of Columbia University, New York, NY, USA
}

Cannabis use disorder (CUD) commonly occurs and carries a notable economic and functional burden at both individual and societal levels. While there are no clearly efficacious medication treatments for CUD, 20 years of committed and high-quality research in the human laboratory and clinical settings have resulted in medications with demonstrated effectiveness in the treatment of cannabis withdrawal, the ability to reduce cannabis use, and results that point to promising future work. The current state of pharmacology research for CUD highlights the need to consider particular characteristics of patients, such as gender, impulsivity, and severity of cannabis use, when selecting a medication in the off-label treatment of CUD or cannabis withdrawal. As a field, the body of work also exposes some areas in need of improvement in study design, selection of outcome measures, interpretation of results, and the overall process of evaluating candidate medications. Coming to a consensus as a field and addressing these gaps in future research will likely lend itself to further advances in improving the lives of patients with CUD.

Neuropsychopharmacology Reviews (20 I8) 43, 173-194; doi: 10.1038/npp.2017.212; published online I8 October 2017

\section{INTRODUCTION}

In terms of large-scale epidemiology, worldwide, cannabis is the most commonly used illicit psychoactive substance, and third overall, coming after alcohol and tobacco (UN Office on Drugs and Crime, 2015). It is estimated that anywhere from 8 to $12 \%$ of regular cannabis users will develop moderate-severe cannabis use disorder (CUD) over time (Moss et al, 2012; Perkonigg et al, 2008). In the United States, 4.2 million people have past-year CUD (SAMHSA, 2015). CUD results in significant impairment and disability (Degenhardt et al, 2013), psychiatric and medical morbidity, poor performance, and legal consequences (Dennis et al, 2002). Fifteen percent of all substance abuse treatment admissions were related to cannabis as the primary, presenting problem in 2014, amounting to roughly 300000 people seeking treatment for a CUD in the United States (SAMHSA, 2016). While this is only a small proportion of the estimated 4.2 million people who have CUD, there is a clear demand for treatment.

Most heavy cannabis users interested in changing their use, are unsuccessful when left to their own devices (Hughes

${ }^{\star}$ Correspondence: Dr CA Brezing, Division on Substance Use Disorders, New York State Psychiatric Institute, 1051 Riverside Drive, Unit 66, New York, NY 10032, USA, Tel: +(646) 774-6132, Fax: +(646) 774-6111, E-mail: brezing@nyspi.columbia.edu

Received 18 April 2017; revised 31 August 2017; accepted 31 August 2017; accepted article preview online 6 September 2017 et al, 2016). Evidence-based psychotherapies have been studied to treat CUD, and various approaches have been shown to have clinical utility (Budney et al, 2000; Budney et al, 2006; Copeland et al, 2001). These include motivational enhancement treatment (MET), cognitive behavioral therapy (CBT), and contingency management (CM), all of which have demonstrated effectiveness in reducing frequency and quantity of cannabis use, but abstinence rates remain modest ( $\sim 20 \%$ continuous abstinence rates) and decline after treatment (Gates et al, 2016). Generally, MET is effective at engaging individuals who are ambivalent about treatment. $\mathrm{CM}$ can lead to longer periods of abstinence during treatment by incentivizing abstinence, and CBT can work to enhance abstinence following treatment (preventing relapse). Longer duration of psychotherapy is associated with improved outcomes (Sherman and McRae-Clark, 2016). Notably, access to evidence-based psychotherapy is frequently limited (Olmstead et al, 2012), and poor adherence to evidence-based psychotherapy by therapists is common (Martino et al, 2009; Santa Ana et al, 2008). Further, implementing $\mathrm{CM}$ techniques, including financial based incentives or vouchers can be challenging and requires a creative and motivated treatment setting. In addition, coverage for psychotherapy from insurance companies is frequently limited both in number of sessions and reimbursement. Because of this, recent interest and promising studies utilizing technology to provide adherent psychotherapy demonstrates a role in the treatment of CUD (Tait et al, 2013). The same 
issues with in person delivery in terms of success rates appear to apply to technologically delivered psychotherapy, in addition to being a nascent field requiring further research and the development of interfaces with larger scale implementation capabilities.

Given the nature of CUD and limitations noted above with psychosocial interventions, effective pharmacotherapy for CUD has been a goal of researchers and clinicians in the field of addiction treatment. Research into pharmacotherapy for CUD has steadily grown from the late 1990s and early 2000 s to the present. Though a relatively new area of research compared to investigations into treatments for other substance use disorders, a number of studies have been completed across a variety of pharmacological targets and a number of reviews on the topic have been previously published (Balter et al, 2014; Gorelick, 2016; Sherman and McRae-Clark, 2016; Vandrey and Haney, 2009). Despite these efforts, to date, there are no FDA-approved medication treatments for CUD. While the completed studies provide valuable information and insight into some potential benefits and clinical utility with regards to the off-label use of medications in specific patient groups and settings, no medication treatment has emerged with enough empirical evidence to provide clear-cut recommendations for treatment.

Here we will present the conceptual framework and rationalizations that have driven the research into possible pharmacotherapy treatment options for CUD, the investigatory models that have provided the data, key limitations of what has been done, a summary of clinical strategies that currently make sense based on the available evidence, and future directions to pursue. For the purposes of this review, we will focus predominantly on placebo-controlled medication trials in the human laboratory and clinical treatment settings for the primary treatment of CUD. We will briefly touch on open-label work that provided the rationale for some of these presented placebo-controlled studies in addition to some work as it is relevant to the treatment of CUD in the context of co-occurring disorders. However, given the scope and breadth of the article and current findings, we will not explore these other areas in great detail.

\section{TWO STUDY MODELS}

All of the placebo-controlled clinical trials exploring candidate medications have occurred in either the (1) human laboratory or (2) the traditional clinical treatment trial. In the former model, participants, who are daily or almost daily cannabis users, many of whom meet criteria for CUD (Pacek and Vandrey, 2014), are brought into the controlled inpatient and/or outpatient laboratory settings. These participants are generally more highly compensated (relative to the treatment trials), which may influence motivations for participation in the studies. In addition, because of the exposure to opportunities to smoke cannabis, participants identify as non-treatment-seeking given the ethical quandary of offering a drug of abuse to individuals attempting to quit or reduce their use. The model of the human laboratory serves as a less costly, more quickly completed, highly controlled environment to trial possible medications as treatments for CUD. Because of the predominantly, within-subject, cross-over designs, a smaller number of participants are needed to achieve the power necessary to identify a signal. Further, the human laboratory has the benefit of identifying important potential interactions between candidate medications and cannabis, in addition to controlling for cannabis use, intoxication, controlled cessation, withdrawal, and relapse. This model postulates that the outcomes would be predictive of those in clinical settings. On the basis of this assumption, negative results would preclude the need to do future expensive and time intensive treatment trials while positive results would identify candidate medications to study further (Haney and Spealman, 2008). The placebo-controlled human laboratory studies of candidate medications for the treatment of CUD are summarized in Table 1.

The latter setting of the clinical treatment trial enrolls individuals meeting Diagnostic and Statistical Manual of Mental Disorders (DSM) criteria for CUD who are seeking treatment (wanting to change their cannabis use) and offering them exposure to medication or placebo that has some reasoning for its potential therapeutic effects on reducing cannabis, though not yet proven efficacy. This setting more closely reflects community-based treatment in physician offices or hospitals, as compared to the human laboratory. This provides the benefit of being more generalizable to the impact a medication may have if utilized in patients' naturalistic settings; however, because fewer factors are controlled for, there are more confounders that may impact results. In addition, participants in this research setting are compensated less relative to the human laboratory participants, so the battery of assessments needs to be more concise so as not to prove too extensive a burden on the individuals' time. Ultimately, candidate medications need to demonstrate positive effects on treating CUD in fully powered trials in this setting to translate into clinical recommendations. The placebo-controlled clinical treatment studies of candidate medications for the treatment of CUD are summarized in Table 2.

\section{CANNABIS WITHDRAWAL-A STARTING POINT}

Despite much earlier laboratory, community, and outpatient study evidence supporting it as a clinically relevant phenomenon, cannabis withdrawal was only first recognized by the DSM in the Fifth Edition (2013) (DSM-V) and adopted by the United States in the International Classification of Diseases Tenth Revision (2015) (ICD-10). The adoption by ICD-10 finally provided a billable (and reimbursable) diagnosis for clinicians. Cannabis withdrawal is defined by DSM-V as having three or more of the following signs and symptoms that develop within 1 week after abrupt reduction or the cessation of prolonged cannabis use: (1) irritability, anger, or aggression; (2) nervousness or 
TABLE 1 Placebo-Controlled Human Laboratory Studies of Medications in Non-treatment-seeking Participants with CUD

\begin{tabular}{lllll}
\hline $\begin{array}{l}\text { Medication and } \\
\text { publication }\end{array}$ & $\begin{array}{l}\text { Number of } \\
\text { participants }\end{array}$ & Participant characteristics & Key study design elements & Outcomes
\end{tabular}

\begin{tabular}{|c|c|c|c|c|}
\hline $\begin{array}{l}\text { Bupropion } 300 \text { mg/day } \\
\text { (divided over two doses) } \\
\text { Haney et al, } 2001\end{array}$ & $N=10$ completers & $\begin{array}{l}\text { Baseline cannabis use: } 6 \pm 1 \text { days/ } \\
\text { week; } 6 \pm 7 \text { joints/day } \\
\text { Gender: } 2 \text { women } \\
\text { Age: } 27 \pm 4 \text { years } \\
\text { Tobacco use: } N=6 \text { cigarette } \\
\text { smokers }\end{array}$ & $\begin{array}{l}\text { Outpatient medication } \\
\text { maintenance with inpatient } \\
\text { phases } \\
\text { Cross-over with PBO, double } \\
\text { blind } \\
\text { Model of steady-state medication } \\
\text { effects on withdrawal }\end{array}$ & $\begin{array}{l}\text { Self-reports of mood VAS, sleep } \\
\text { VAS, food intake, psychomotor task } \\
\text { performance }\end{array}$ \\
\hline $\begin{array}{l}\text { Nefazodone } 450 \text { mg/day } \\
\text { (divided over two doses) } \\
\text { Haney et al, } 2003\end{array}$ & $\begin{array}{l}N=7 \text { completers ( } 4 \\
\text { additional volunteers } \\
\text { were not included) }\end{array}$ & $\begin{array}{l}\text { Baseline cannabis use: } 6.4 \pm 0.4 \text { days/ } \\
\text { week; } 6 \pm 1.3 \text { joints/day } \\
\text { Gender. I woman } \\
\text { Age: } 30 \pm 3 \text { years }\end{array}$ & $\begin{array}{l}\text { Outpatient medication phases } \\
\text { Cross-over with PBO, double } \\
\text { blind } \\
\text { Model of steady-state medication }\end{array}$ & $\begin{array}{l}\text { Self-reports of mood VAS, sleep } \\
\text { VAS, food intake, psychomotor task } \\
\text { performance }\end{array}$ \\
\hline
\end{tabular}

$\begin{array}{lll}\text { Mirtazapine } 30 \mathrm{mg} \text { nightly } & \mathrm{N}=1 \mathrm{I} \text { completers (I } & \text { Baseline cannabis use: } 6.9 \pm 0.2 \text { days/ } \\ \text { additional volunteer } & \text { week; II.9 } \pm 5.3 \text { joints/day } \\ \text { dropped out) } & \text { Gender: } 0 \text { women } \\ & \text { Age: } 27 \pm 5 \text { years } \\ & \text { Tobacco use: } N=7 \text { cigarette } \\ & \text { smokers }\end{array}$

Divalproex $1500 \mathrm{mg} /$ day (divided over two doses) Haney et al, 2004
$N=7$ completers $(1$ additional volunteer dropped out)

\section{Baseline cannabis use: $6.6 \pm 0.3$ days/} week; $6.3 \pm 2$ joints/day Gender: I women

Age: $26 \pm 1$ years

Tobacco use: $N=4$ cigarette smokers

$N=14$ completers $(6$ additional volunteers dropped out) week; $10.0 \pm 6.5$ joints/day

Gender. 2 women

\section{Baseline cannabis use: $6.6 \pm 0.9$ days}

Age: $26 \pm 4$ years

Tobacco use: $N=10$ cigarette smokers using $5.7 \pm 3.7$ cigarettes/day

Baclofen 60 and $90 \mathrm{mg} /$ day (divided over three doses)

Haney et al, 2010

$N=10$ completers $(3$ additional volunteers dropped out)

Baseline cannabis use: $6.7 \pm 0.9$ days/ week; $9.4 \pm 3.9$ joints/day

Gender: 0 women

Age: $29 \pm 6$ years
Tobacco use: $N=8$ cigarette smokers

$\begin{array}{ll}\text { Zolpidem } 12.5 \mathrm{mg} \text { nightly } & \mathrm{N}=20 \text { completers ( } 5 \\ \text { Vandrey et al, 2011 } & \begin{array}{l}\text { additional volunteers } \\ \text { dropped out) }\end{array}\end{array}$

Dronabinol 50 mg/day (divided in five doses) Haney et al, 2004
$N=7$ completers $(4$ additionally volunteers dropped out)

\section{Baseline cannabis use: daily cannabis} smokers who smoked up to $4 \times 1$ day \pm 3 times

Gender: 3 women

Age: $29 \pm 8$ years

Tobacco use: $N=16$ cigarette smokers smoked $9 \pm 6$ times/day

\section{Baseline cannabis use: $6.2 \pm 1.3$ days/} week; $9.6 \pm 6.2$ joints/day

Gender: 0 women
Age: $24 \pm 1$ years

Tobacco use: $N=6$ cigarettes smokers

\begin{tabular}{|c|c|c|}
\hline $\begin{array}{l}\text { Dronabinol } 30 \text { or } 90 \mathrm{mg} / \\
\text { day (divided in three } \\
\text { doses) } \\
\text { Budney et al, } 2007\end{array}$ & $\begin{array}{l}N=8 \text { completers ( } 14 \\
\text { additional non- } \\
\text { completers) }\end{array}$ & $\begin{array}{l}\text { Baseline cannabis use: } 28.5 \pm 1.9 \text { days } \\
\text { of cannabis use/ } 30 \text { days; using } \\
2.6 \pm 0.5 \text { times/day } \\
\text { Gender: } 2 \text { women } \\
\text { Age: } 32.5 \text { years }(21-54 \text { years) } \\
\text { Tobacco use: } N=4 \text { cigarette } \\
\text { smokers }\end{array}$ \\
\hline
\end{tabular}

Dronabinol $60 \mathrm{mg} / \mathrm{day}$ +lofexidine $2.4 \mathrm{mg} /$ day (divided by four doses) Haney et al, 2008

\section{$N=8$ completers}

Baseline cannabis use: daily cannabis users; $12.2 \pm 8.1$ joints/day Gender: 0 women

Age: $29 \pm 7$ years

Tobacco use: $N=6$ cigarette smokers; $9.3 \pm 5.9$ cigarettes/day

effects on withdrawal

\section{Outpatient medication} maintenance with inpatient phases

Cross-over with PBO, double blind

Model of medication effects on behavior, withdrawal, and relapse to marijuana smoking

Outpatient medication maintenance with inpatient phases

Cross-over design with PBO, double blind

Model of medication effects on behavior and withdrawal

Inpatient phases

Cross-over, double blind

Model of medication's effects on

withdrawal and relapse prevention

Inpatient phases

Cross-over, double blind Model of medication effects on behavior, withdrawal, and relapse to marijuana smoking

\section{Outpatient and inpatient phases}

Cross-over design with $\mathrm{PBO}$

Model of natural cannabis withdrawal effects on sleep and zolpidem's effects on sleep in the context of withdrawal

Outpatient medication maintenance with inpatient sessions

Cross-over design with $\mathrm{PBO}$ double blind

Model of medication effects on behavior and withdrawal

\section{Outpatient study; multiple} sessions

Cross-over with PBO, double blind

Model of medication's effects on withdrawal symptoms in more naturalistic setting (as compared to inpatient)

Inpatient phases

Cross-over with four arms, double blind

Model of medication's effects on withdrawal and relapse prevention
Self-reports of mood VAS, sleep VAS, subjective effects VAS, craving VAS, food intake, psychomotor task performance, Actiwatch data, mean money spent to purchase marijuana following abstinence

Self-reports of mood VAS, sleep VAS, subjective effects VAS, MWC (including craving), food intake and weight, psychomotor task performance, social task

Nightcap system data on sleep

Self-reports of VAS on affective and physical symptoms, sleep VAS, Actiwatch, psychomotor task battery, food intake, mean money spent to purchase marijuana following abstinence

Self-reports of mood VAS, sleep VAS, subjective effects VAS, craving VAS, food intake, psychomotor task performance, Actiwatch data, mean money spent to purchase marijuana following abstinence

PSG

PSQI

MWC

MCQ

Side effects

Cognitive performance battery

Self-reports of mood VAS, sleep VAS, subjective effects VAS, drug effects, MWC (including craving), food intake and weight, psychomotor task performance, social task

Nightcap system data on sleep

MWC

MCQ

$\mathrm{BSI}$

POMS

Sleep self-report

ARCl

Drug effects VAS

Side effects

Subjective effects VAS, mood VAS, craving VAS, observer ratings, food intake and weight, psychomotor task performance, social task, sleep VAS, blood pressure, nightcap system data on sleep, mean money spent to purchase marijuana following abstinence 
Table 1 (Continued)

\begin{tabular}{|c|c|c|c|c|}
\hline $\begin{array}{l}\text { Medication and } \\
\text { publication }\end{array}$ & $\begin{array}{l}\text { Number of } \\
\text { participants }\end{array}$ & Participant characteristics & Key study design elements & Outcomes \\
\hline $\begin{array}{l}\text { Nabilone } 6 \text { and } 8 \mathrm{mg} / \text { day } \\
\text { (divided in two doses) } \\
\text { Haney, 2013b }\end{array}$ & $\begin{array}{l}N=\mid \text { I completers ( } \\
\text { additional participant } \\
\text { dropped out) }\end{array}$ & $\begin{array}{l}\text { Baseline cannabis use: } 6.9 \pm 0.3 \text { days/ } \\
\text { week; } 8.3 \pm 3.1 \text { joints/day } \\
\text { Gender: } 3 \text { women } \\
\text { Age: } 30 \pm 10 \text { years } \\
\text { Tobacco use: } N=11 \text { cigarettes } \\
\text { smokers; } 5.7 \pm 5.5 \text { cigarettes/day }\end{array}$ & $\begin{array}{l}\text { Inpatient phases } \\
\text { Cross-over with PBO, double } \\
\text { blind } \\
\text { Model of medication's effects on } \\
\text { withdrawal and relapse } \\
\text { prevention }\end{array}$ & $\begin{array}{l}\text { Self-reports of mood VAS, sleep } \\
\text { VAS, subjective effects VAS, craving } \\
\text { VAS, food intake, psychomotor task } \\
\text { performance, Actiwatch data, mean } \\
\text { money spent to purchase marijuana } \\
\text { following abstinence }\end{array}$ \\
\hline $\begin{array}{l}\text { Nabilone } 6 \mathrm{mg} / \text { day } \\
\text { +zolpidem } 12.5 \mathrm{mg} \text { nightly } \\
\text { (divided by three doses) } \\
\text { Herrmann et al, } 2016\end{array}$ & $\begin{array}{l}\mathrm{N}=\text { I I completers ( } 4 \\
\text { additional volunteers } \\
\text { dropped out) }\end{array}$ & $\begin{array}{l}\text { Baseline cannabis use: } 6.8 \pm 0.4 \text { days/ } \\
\text { week; } 9.6 \pm 4.6 \text { joints/day } \\
\text { Gender: } 0 \text { women } \\
\text { Age: } 27.5 \pm 6.1 \text { years } \\
\text { Tobacco use: } N=9 \text { cigarette } \\
\text { smokers; } 3.9 \pm 2.3 \text { cigarettes/day }\end{array}$ & $\begin{array}{l}\text { Inpatient phases } \\
\text { Cross-over with three arms, } \\
\text { double blind } \\
\text { Model of medication's effects on } \\
\text { withdrawal and relapse } \\
\text { prevention }\end{array}$ & $\begin{array}{l}\text { Self-reports of mood VAS, sleep } \\
\text { VAS, subjective effects VAS capsule, } \\
\text { craving VAS, food intake and body } \\
\text { weight, cognitive task performance, } \\
\text { Actiwatch data, mean money spent } \\
\text { to purchase marijuana following } \\
\text { abstinence }\end{array}$ \\
\hline $\begin{array}{l}\text { Cannabidiol } 20,400 \text {, and } \\
800 \mathrm{mg} \text { I } \times \text { dose } \\
\text { Haney et al, } 2016\end{array}$ & $\begin{array}{l}N=31 \text { completers } \\
\text { (19 additional } \\
\text { volunteers dropped } \\
\text { out) }\end{array}$ & $\begin{array}{l}\text { Baseline cannabis use: } 6.5 \pm 1.0 \text { days/ } \\
\text { week; } 5.1 \pm 5.3 \text { joints/day } \\
\text { Gender: } 14 \text { women } \\
\text { Age: } 29.1 \pm 9.1 \text { years } \\
\text { Tobacco use: } N=18 \text { cigarette } \\
\text { smokers }\end{array}$ & $\begin{array}{l}\text { Outpatient study, multiple } \\
\text { sessions } \\
\text { Within subject, double blind } \\
\text { Model of medication's subjective } \\
\text { and physiological effects and self- } \\
\text { administration of marijuana }\end{array}$ & $\begin{array}{l}\text { Self-reports of mood VAS, sleep } \\
\text { VAS, subjective effects VAS, drug } \\
\text { effects VAS, and marijuana effects } \\
\text { VAS, capsule VAS, performance task } \\
\text { battery, HR, BP, plasma CBD }\end{array}$ \\
\hline $\begin{array}{l}\text { Rimonabant I, 3, 10,30, or } \\
90 \mathrm{mg} \text { I × dose } \\
\text { Huestis et al, 200 I }\end{array}$ & $\begin{array}{l}N=63 \text { randomized } \\
\text { participants ( } / \text { group } \\
\text { range from } 6 \text { to } 10 \\
\text { participants/group) }\end{array}$ & $\begin{array}{l}\text { Baseline cannabis use: } 4.6-25.0 \text { days/ } \\
30 \text { days } \\
\text { Gender: } 0 \text { women } \\
\text { Age: } 21-45 \text { years }\end{array}$ & $\begin{array}{l}\text { Inpatient phase } \\
\text { Participants randomized to one } \\
\text { of eight groups (combinations of } \\
\text { PBO medication, escalating } \\
\text { doses of rimonabant, active } \\
\text { cannabis, PBO cannabis) } \\
\text { Model of medication's dose } \\
\text { effects on physiological and } \\
\text { subjective effects of smoked } \\
\text { cannabis }\end{array}$ & $\begin{array}{l}\text { HR } \\
\text { Subjective effects of smoked cannabis } \\
\text { VAS } \\
\text { ARCI } \\
\text { Plasma cannabinoid concentrations } \\
\text { Plasma rimonabant concentrations }\end{array}$ \\
\hline $\begin{array}{l}\text { Rimonabant } 40 \text { mg/day or } \\
90 \mathrm{mg} \text { I } \times \text { dose } \\
\text { Huestis et al, } 2007\end{array}$ & $\begin{array}{l}N=36 \text { completers; } \\
N=12 \text { in each arm ( } 6 \\
\text { additional volunteers } \\
\text { dropped out) }\end{array}$ & $\begin{array}{l}\text { Baseline cannabis use: } \\
20.3 \pm 9.6 \text { days/last } 30 \text { days } \\
\text { Gender: } 0 \text { women } \\
\text { Age: } 28.9 \pm 6.3 \text { years }\end{array}$ & $\begin{array}{l}\text { Inpatient phase } \\
\text { Randomized, double-blind, } \\
\text { parallel group design of three } \\
\text { groups (maintenance } \\
\text { rimonabant, } \mathrm{PBO}+\text { I high-dose } \\
\text { rimonabant, or PBO) } \\
\text { Model of medication's effects on } \\
\text { physiological and subjective } \\
\text { effects of smoked cannabis }\end{array}$ & $\begin{array}{l}\text { HR } \\
\text { Subjective effects of smoked cannabis } \\
\text { VAS } \\
\text { ARCI } \\
\text { Plasma cannabinoid } \\
\text { concentrations } \\
\text { Plasma rimonabant concentrations }\end{array}$ \\
\hline $\begin{array}{l}\text { Naltrexone } 12,25,50 \text {, and } \\
100 \mathrm{mg} I \times \text { dose } \\
\text { Cooper and Haney, } 2010\end{array}$ & $\begin{array}{l}N=29 \text { completers } \\
\text { (20 additional } \\
\text { participants dropped } \\
\text { out) }\end{array}$ & $\begin{array}{l}\text { Baseline cannabis use: } 6.7 \pm 0.1 \text { days/ } \\
\text { week; } 8.3 \pm 1.2 \text { joints/day; } \\
\$ 83.2 \pm 13.1 / \text { week spent on cannabis } \\
\text { Gender: } 14 \text { women } \\
\text { Age: } 28 \pm \text { I years } \\
\text { Tobacco use: } N=24 \text { cigarette } \\
\text { smokers; } 6.8 \pm 0.9 \text { cigarettes/day }\end{array}$ & $\begin{array}{l}\text { Outpatient study, multiple } \\
\text { sessions } \\
\text { Within subject, double blind } \\
\text { Model of medications' dose } \\
\text { effects on subjective, } \\
\text { physiological, and cognitive } \\
\text { effects of smoked cannabis }\end{array}$ & $\begin{array}{l}\text { Subjective effects VAS, drug effects } \\
\text { VAS, and marijuana effects VAS, } \\
\text { capsule VAS, cognitive task battery, } \\
\text { HR, BP }\end{array}$ \\
\hline $\begin{array}{l}\text { Naltrexone } 50 \mathrm{mg} / \text { day } \\
\text { Haney et al, } 2015\end{array}$ & $\begin{array}{l}N=51 \text { randomized } \\
\text { completers ( } N=23 \\
\text { on naltrexone; } 17 \\
\text { additional volunteers } \\
\text { dropped out; } 9 \text { on } \\
\text { naltrexone) }\end{array}$ & $\begin{array}{l}\text { Baseline cannabis use: } \mathrm{PBO} \text {, smoked } \\
6.3 \pm 1 \text { days/week; } 6.0 \pm 4.0 \text { joints/ } \\
\text { day; naltrexone, } 6.4 \pm 1 \text { days/week; } \\
5.5 \pm 3.6 \text { joints/day } \\
\text { Gender: } 7 \text { women ( } N=5 \text { on } \\
\text { naltrexone) } \\
\text { Age: } P B O, 31.6 \pm 8 \text { years; naltrexone: } \\
28.6 \pm 7 \text { years }\end{array}$ & $\begin{array}{l}\text { Outpatient study, multiple } \\
\text { sessions } \\
\text { Randomized to PBO or } \\
\text { naltrexone given as maintenance } \\
\text { dosing over I } 6 \text { days } \\
\text { Model of medication's effects on } \\
\text { self-administration of cannabis }\end{array}$ & $\begin{array}{l}\text { Self-reports of mood VAS, sleep } \\
\text { VAS, subjective effects VAS, drug } \\
\text { effects VAS, and marijuana effects } \\
\text { VAS, capsule VAS, cognitive task } \\
\text { battery, HR, BP, plasma naltrexone }\end{array}$ \\
\hline
\end{tabular}

Abbreviations: ARCl, Addiction Resource Center Inventory; BP, blood pressure; BSI, Brief Symptom Inventory; HR, heart rate; MCQ, Marijuana Craving Questionnaire; MWC, Marijuana Withdrawal Checklist; PBO, placebo; POMS, profile of mood states; PSG, polysomnography; PSQI, Pittsburgh Sleep Quality Inventory; VAS, Visual Analog Scale.

Numbers provided in mean $\pm \mathrm{SD}$.

Note that if the numbers for dropouts, baseline cannabis use, or tobacco use are not provided, they were not presented in the primary article.

anxiety; (3) sleep difficulty (eg, insomnia or vivid dreaming); (4) decreased appetite or weight loss; (5) restlessness; (6) depressed mood; and (7) at least one of the following physical symptoms that causes discomfort: abdominal pain, shakiness/tremors, sweating, fever, chills, or headache (APA, 2013). Notably, the DSM-V does not include craving as a symptom of cannabis withdrawal, though this particular symptom has generally been identified as an important factor in CUD, cannabis withdrawal, and is a common outcome measure in CUD treatment trials. We know that cannabis withdrawal symptoms may be present within the first $24 \mathrm{~h}$ and overall peak within the first week, lasting up to1 month following last use (Bonnet and Preuss, 2017). Biologically there is evidence that cannabis withdrawal is 
associated with the downregulation of brain cannabinoid-1 (CB-1) receptors as a result of chronic use. When cessation (or relative cessation) of activity at $\mathrm{CB}-1$ receptors occurs, the deficiency results in interference with other neurotransmitter systems, leading to the above-mentioned symptoms (Bonnet and Preuss, 2017).

Cannabis withdrawal is clinically significant because of the unpleasant symptoms that can interfere with daily functioning in addition to serving as a negative reinforcer driving relapse and ongoing use of cannabis (Allsop et al, 2012). Research in the treatment of CUD began in the human laboratory by selecting pharmacological agents that would improve specific signs or symptoms and/or attenuate cannabis withdrawal more globally when cannabis cessation was induced in the lab. Although this is the rationale utilized by many of the clinical treatment studies, this has not been as well defined in the design and assessments in this setting. Measures, such as the Cannabis Withdrawal Scale, which synthesized all of the previously available scales and provided a validated checklist of symptoms (Allsop et al, 2011), is not routinely used to measure cannabis withdrawal in the treatment trials.

Withdrawal was a good starting point for the assessment of medications for the treatment of CUD and helpful to treat in and of itself for the comfort of patients abstaining or reducing cannabis use. However, it appears based on the available evidence to date that for most patients medications that are effective in treating withdrawal are likely not sufficient as monotherapies at reducing cannabis use or resulting in abstinence. Here we can take a lesson from tobacco-smoking cessation, as nicotine replacement therapy (NRT) is generally not sufficient as a monotherapy for most patients trying to achieve smoking cessation and the combination of other pharmacological agents (eg, bupropion or varenicline) with NRT in conjunction with counseling is now the gold standard of treatment (Stead et al, 2008; Mills et al, 2012).

\section{NON-CANNABINOID MEDICATIONS}

\section{Noradrenergic Agents}

Some of the earliest work investigating medication as a potential treatment for CUD looked at antidepressants with noradrenergic reuptake inhibition. At the time, Budney et al (2008) demonstrated that there was notable overlap in the phenotypes of cannabis withdrawal and nicotine withdrawal, including irritability, physical tension, anxiety, insomnia, and low/depressed mood (Budney et al, 2008; Vandrey et al, 2008). Sustained release bupropion (Zyban), an atypical antidepressant in that it mainly inhibits the reuptake of norepinephrine and dopamine with limited action on serotonin, had recently been approved by the FDA (1997) for tobacco-smoking cessation, prompting interest in its potential for the treatment for CUD. Clinically, bupropion is considered a more 'activating' atypical antidepressant and is commonly utilized in the off-label treatment of ADHD when stimulants are not recommended.

Haney et al (2001) first looked at sustained release bupropion's effects on cannabis withdrawal. The study made use of a combination outpatient maintenance medication phase and inpatient crossover design. Participants were nontreatment-seeking heavy cannabis users who were initially maintained on either double-blinded sustained release bupropion or placebo. These participants smoked active marijuana for the initial part of the inpatient phase followed by placebo marijuana (no $\Delta$-9-tetrahydrocannabinol; THC) to precipitate withdrawal from cannabis. Sustained release bupropion had few effects when participants smoked active marijuana; however, during the placebo marijuana phase, compared to placebo medication, participants had worse ratings of irritability, restlessness, depression (feeling miserable), and trouble sleeping on sustained release bupropion. It was concluded that bupropion exacerbates cannabis withdrawal and would have no utility in the treatment of CUD (Haney et al, 2001).

In a similarly designed human laboratory study in the same participant population, Haney et al (2003) looked at nefazodone as a candidate medication. Nefazodone is an antidepressant that similarly to bupropion functions as a norepinephrine reuptake inhibitor in addition to having functions as a serotonin reuptake inhibitor and serotonin $2 \mathrm{~A}$ receptor antagonist effects. Nefazodone was selected because of previous findings of its impact on reductions in cravings in a different drug of abuse (cocaine) (Kampman et al, 1999) and its clinical effects on improving sleep and anxiety. In the human lab study, nefazodone reduced anxiety and muscle pain during cannabis withdrawal but had no impact on sleep disturbances or irritability. The study concluded that nefazodone likely did not have a role in the treatment of CUD (Haney et al, 2003).

Following these two human laboratory studies, Carpenter et al (2009) completed a clinical trial of either sustained release bupropion or nefazodone $v s$ placebo for CUD in conjunction with coping skills therapy. The trial was a 13week outpatient study of treatment-seeking individuals with cannabis dependence by DSM-IV. Goals of the study were to assess these two medications in the treatment of cannabis withdrawal and overall maintenance treatment for CUD (Carpenter et al, 2009). Given the two human laboratory studies completed by Haney et al (2001, 2003) that were negative, the rationale was that the differences in setting (inpatient human laboratory vs outpatient clinic setting), patient population (treatment-seeking $v s$ non-treatmentseeking), and dose limitations (fixed $v s$ adjustable to patient side effects; subacute $v s$ chronic), warranted a larger clinical trial follow-up from the laboratory studies. Notable findings included no change in cannabis use severity (as measured by CGI) or change in symptoms of sleep, irritability, or anxiety in either active medication arm compared to placebo. Notable problems included high dropout rates (only 49\% completed medication and only $43 \%$ completed the entire trial), leading to a small number of participants in each arm, 
TABLE 2 Placebo-Controlled Clinical Treatment Studies of Medications in Treatment-seeking Participants with CUD

\begin{tabular}{|c|c|c|c|c|}
\hline $\begin{array}{l}\text { Intervention and } \\
\text { publication }\end{array}$ & Number of participants & Participant characteristics & Key study design elements & Outcomes \\
\hline $\begin{array}{l}\text { Bupropion SR I50 mg b. } \\
\text { i.d.+weekly individual } \\
\text { coping skill sessions } \\
\text { Carpenter et al, } 2009\end{array}$ & $\begin{array}{l}N=106 \text { randomized; } 40 \text { in } \\
\text { bupropion } \\
N=66 \text { completed } 6 \text { weeks } \\
\text { of study } \\
N=52 \text { completed } 10 \text {-week } \\
\text { medication phase (with } 21 \\
\text { on bupropion) } \\
N=46 \text { completed } 13 \text { weeks } \\
\text { (with } N=18 \text { on bupropion) }\end{array}$ & $\begin{array}{l}\text { Baseline cannabis use: } 29 / 30 \text { days } \\
\text { Age: } 32 \pm 10 \text { years } \\
\text { Gender: } 25 \text { women randomized } \\
\text { Cigarettes/nicotine use not } \\
\text { reported }\end{array}$ & $\begin{array}{l}\text { I } 3 \text { weeks; I } 0 \text {-week active } \\
\text { medication; I-week PBO lead-in; } \\
\text { 2-week PBO lead-out } \\
\text { Outpatient setting } \\
\text { Double blind } \\
\text { Three arms (bupropion, } \\
\text { nefazodone, or PBO) }\end{array}$ & $\begin{array}{l}{ }^{\text {a}} \text { Self-report of cannabis in grams } \\
\text { and dollar amount } \\
{ }^{a} \text { Frequency of days used } \\
{ }^{a} \text { Qualitative THC-COOH cutoff } \\
\text { CGl-cannabis use severity } \\
\text { Self-report scales of sleep } \\
\text { (SMHSQ) and irritability (Snaith) } \\
\text { Clinician-administered anxiety scale } \\
\text { (HAM-A) } \\
\text { Riboflavin for adherence } \\
\text { Side effects }\end{array}$ \\
\hline $\begin{array}{l}\text { Bupropion SR I } 50 \text { mg b. } \\
\text { i.d.+weekly MET } \\
\text { Penetar et al, } 2012\end{array}$ & $\begin{array}{l}N=22 \text { randomized; } 9 \\
\text { completed the study ( } 5 \text { in } \\
\text { bupropion; } 4 \text { in PBO) }\end{array}$ & $\begin{array}{l}\text { Baseline cannabis use: daily } \\
\text { cannabis users } \\
\text { Age: } 31.2 \pm 9.6 \text { years } \\
\text { Gender: } 4 \text { women completed } \\
\text { study } \\
\text { Tobacco use: } N=2 \text { cigarette } \\
\text { smokers; smoked }<5 \text { cigarettes/ } \\
\text { day }\end{array}$ & $\begin{array}{l}2 \text { I days } \\
\text { Outpatient setting; time-intensive } \\
\text { daily study visits } \\
\text { Double blind } \\
\text { Medication lead-in with target } \\
\text { quit day (day 8) }\end{array}$ & 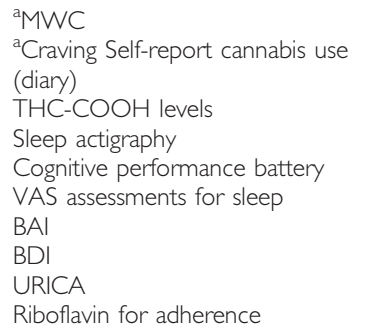 \\
\hline
\end{tabular}

Nefazodone $300 \mathrm{mg}$ b.i.d.+weekly individual coping skill sessions Carpenter et al, 2009
$N=106$ randomized; 36 in nefazodone

$N=66$ completed 6 weeks of study

$N=52$ completed 10 -week medication phase (with 15 on nefazodone)

$N=46$ completed 13 weeks (with $N=14$ on

nefazodone)

Atomoxetine $100 \mathrm{mg}$ daily +3 sessions of MET McRae-Clark et al, 2010

$N=78$ randomized (with 39 in each group)

Full ITT sample of $N=46$ Modified ITT sample of 38 participants $(N=19$ in each group) who returned for at least one post-baseline assessment

$N=16$ completed 12 weeks

Venlafaxine-XR $225 \mathrm{mg}$ daily+weekly CBT/RPT Levin et al, 2013

\section{$N=103$ randomized (with}

$N=5 I$ in venlafaxine)

$N=87$ completed 6 weeks of treatment (with $N=40$ in venlafaxine) $N=64$ completed 12 weeks (with 31 in venlafaxine)
Baseline cannabis use: $27 / 30$ days Age: $32 \pm 10$ years

Gender: 25 women randomized Cigarettes/nicotine use not reported
I3 weeks; 10-week active medication; I-week PBO lead-in; 2-week PBO lead-out Outpatient setting

Double blind Three arms (bupropion or PBO)

aself-report of cannabis in grams and dollar amount a Frequency of days used ${ }^{a} Q$ ualitative urine THC $\mathrm{COOH}$ cutoff

CGI-cannabis use severity

Self-report scales of sleep (SMHSQ) and irritability (Snaith) Clinician-administered anxiety scale (HAM-A)

Riboflavin

Side effects

Baseline cannabis use: used $86.8 \%$ ( $\pm 22.8 \%$ ) days on TLFB-90d all

with co-occurring ADHD

Age: $29.9 \pm 11.5$ years

Gender: 9 women randomized in modified ITT

Cigarettes/nicotine use not

reported

12 weeks; medication titrated to maintenance by fourth week

Outpatient setting

Double blind

Flex medication dosing

TLFB in week 12

aTLFB throughout study $\mathrm{MCQ}$

aSemi-quantitative UDS 2CGI-I

ADHD and cannabis

CGI-S ADHD and Cannabis

HAM-A

HAM-D

2WRAADS

CAARS-self

Side effects

Baseline cannabis use: $27.4 / 30$ days

all with co-occurring depression

12 weeks; I-week PBO lead-in;

a 2 consecutive weeks abstinence by TLFB

8-week medication maintenance

Age: $35.9 \pm 9.3$ years $(\mathrm{PBO})$ and

$34.2 \pm 10.8$ (venlafaxine)

Gender: 27 women (out of 103

Outpatient setting

Double blind

Flexible medication dosing

randomized)

Cigarettes/nicotine use not reported

${ }^{a}$ Quantitative urine $\mathrm{THC}-\mathrm{COOH}$ levels

aHAM-D

Longitudinal cannabis outcomes Side effects

Treatment adherence

Relationship of cannabis to depression

Buspirone $30 \mathrm{mg}$ b.i.d

+3 sessions of MET

$N=93$ randomized $(N=49$

in buspirone)

Full ITT sample of $N=59$

Modified ITT sample of 50 participants $(N=23$ in buspirone) who returned for at least one post-baseline assessment

$N=1 \mid$ completed 12 weeks

Buspirone $30 \mathrm{mg}$ bi.d.

+3 sessions MET

McRae-Clark et al, 2015

$N=175$ randomized

( $N=88$ on buspirone)

$N=146$ received at least $\mid$

post-baseline assessment

( $N=77$ in buspirone)

$N=92$ completers $(N=45$

on buspirone)
Used cannabis $89 \%( \pm 16 \%)$ days
on TLFB-90d; 3.8 joints/day $( \pm 2.5)$

Age: $31.6 \pm 10.2$ years

Gender: 5 women in modified ITT

(out of 50)

Cigarettes/nicotine use not

reported

12 weeks; medication titrated as tolerated

Outpatient setting

Double blind

Proportion of weekly negative

UDS time to first negative UCT

TLFB (\%days abstinent; amount

used per day)

$\mathrm{MCQ}$

MWC

HAM-A
Used cannabis $85.2 \%$ of days (90-d

TLFB); 3.8 joints/day

Age: 24 years $(23.1-25)$

Gender: 41 women (out of 175

randomized)

Cigarettes/nicotine use not

reported
12 weeks: medication titrated as tolerated

Outpatient setting

Double blind

Mean dose of

medication $=42 \mathrm{mg} /$ day; twice

daily dosing aproportion of weekly negative

UCT

${ }^{\mathrm{M}} \mathrm{MCQ}$

2Gender

5 HTIA-R genotype HAM-A

Semi-quantitative UDS

Study retention

Medication adherence

Side effects 
Table 2 (Continued)

\begin{tabular}{|c|c|c|c|c|}
\hline $\begin{array}{l}\text { Intervention and } \\
\text { publication }\end{array}$ & Number of participants & Participant characteristics & Key study design elements & Outcomes \\
\hline $\begin{array}{l}\text { Escitalopram } 10 \text { mg/day } \\
\text { +9 sessions CBT/RPT } \\
\text { Weinstein et al, } 2014\end{array}$ & $\begin{array}{l}N=52 \text { randomized }(N=26 \\
\text { on escitalopram) } \\
N=26 \text { completers }(N=10 \\
\text { on citalopram) }\end{array}$ & $\begin{array}{l}\text { Baseline cannabis use not reported } \\
\text { Age: } 32 \pm 6.8 \text { years } \\
\text { Gender. } 13 \text { women } \\
\text { Cigarettes/nicotine use not } \\
\text { reported }\end{array}$ & $\begin{array}{l}\text { I0 weeks; I-week PBO lead in; } \\
\text { 9-week fixed dose escitalopram } \\
\text { vs PBO } \\
\text { Outpatient treatment setting } \\
\text { 6-month follow-up post } \\
\text { medication }\end{array}$ & $\begin{array}{l}{ }^{a} \text { Qualitative urine for } \mathrm{THC}-\mathrm{COOH} \\
\text { STAI } \\
\text { BDI } \\
\text { CIWA for cannabis }\end{array}$ \\
\hline $\begin{array}{l}\text { Vilazodone } 40 \text { mg/day } \\
+3 \text { sessions MET } \\
\text { McRae-Clark et al, } 2016\end{array}$ & $\begin{array}{l}N=76 \text { randomized }(N=4 \mid \\
\text { on vilazodone) } \\
N=59 \text { received at least I } \\
\text { post-baseline assessment } \\
(N=28 \text { vilazodone) } \\
N=31 \text { completers }(N=14 \\
\text { vilazodone) }\end{array}$ & $\begin{array}{l}\text { Baseline cannabis use: } 81.9 \% \text { of } \\
\text { days ( } 90-d \text { TLFB); } 3.3 \text { joints/day } \\
\text { Age: } 22 \text { years (21.3-23.I) } \\
\text { Gender: } 16 \text { women (out of } 76 \\
\text { randomized) } \\
\text { Cigarettes/nicotine use not } \\
\text { reported }\end{array}$ & $\begin{array}{l}8 \text { weeks; medication titrated as } \\
\text { tolerated } \\
\text { Outpatient treatment setting } \\
\text { Double blind } \\
\text { Mean dose of } \\
\text { medication = } 32 \mathrm{mg} / \text { day; once } \\
\text { daily dosing }\end{array}$ & $\begin{array}{l}\text { aUCT } \\
\text { a Weekly TLFB } \\
\text { MCQ } \\
\text { HAM-A } \\
\text { HAM-D }\end{array}$ \\
\hline $\begin{array}{l}\text { Divalproex } 750- \\
1000 \text { mg b.i.d.+weekly } \\
\text { CBT/RPT } \\
\text { Levin et al, } 2004\end{array}$ & $\begin{array}{l}N=25 \text { randomized }(N=13 \\
\text { on divalproex }) \\
N=9 \text { completers }(N=5 \text { on } \\
\text { divalproex })\end{array}$ & $\begin{array}{l}\text { Baseline cannabis use: } \\
6.8 \pm 0.6 \text { days/week; } 28.3 \pm 23.2 \\
\text { joints/week } \\
\text { Age: } 31.5 \pm 4.9 \text { years } \\
\text { Gender: } 2 \text { women (out of } 25 \\
\text { randomized) } \\
\text { Cigarettes/nicotine use not } \\
\text { reported }\end{array}$ & $\begin{array}{l}\text { I4-week study; 2-week PBO } \\
\text { lead-in followed by cross-over of } \\
6 \text { weeks of active medication and } \\
6 \text { weeks of PBO } \\
\text { Medication titrated to } 2000 \mathrm{mg} / \\
\text { day as tolerated and based on } \\
\text { blood levels of active medication } \\
\text { Double blind } \\
\text { Outpatient setting }\end{array}$ & $\begin{array}{l}{ }^{\text {a}} \text { Retention of participants in } \\
\text { treatment } \\
\text { Self-report of frequency and } \\
\text { amount of cannabis used } \\
\text { VAS for craving } \\
\text { Semi-quantitative UCT CGI } \\
\text { cannabis use severity } \\
\text { Irritability (Snaith and Hopkins } \\
\text { Symptom checklist) } \\
\text { Side effects }\end{array}$ \\
\hline $\begin{array}{l}\text { Lithium carbonate } \\
500 \text { mg b.i.d. } \\
\text { Johnston et al, } 2014\end{array}$ & $\begin{array}{l}N=4 \mid \text { randomized }(N=19 \\
\text { on lithium) } \\
N=38 \text { received }>\text { I dose of } \\
\text { medication ( } N=16 \text { on } \\
\text { lithium) } \\
N=17 \text { stayed inpatient } \\
(N=8 \text { on lithium) } \\
N=29 \text { out of } 38 \text { who } \\
\text { received medication at } \\
\text { I } 4 \text { days follow up ( } N=13 \\
\text { on lithium) } \\
N=25 \text { at } 30 \text { days' follow-up } \\
(N=15 \text { on lithium) } \\
N=26 \text { at } 90 \text { days' follow-up } \\
(N=13 \text { on lithium) }\end{array}$ & $\begin{array}{l}\text { Baseline cannabis use: } 7 / 7 \text { days/ } \\
\text { week; used } 3.82 \pm 2.79 \text { g/day } \\
\text { Age: } 40.51 \pm 12.49 \\
\text { Gender: } 13 \text { women (out of } 38 \text { ) } \\
\text { Tobacco use: } 69-75 \% \text { were almost } \\
\text { daily tobacco users }\end{array}$ & $\begin{array}{l}8 \text { days/7 nights inpatient } \\
\text { admission with } 14-, 30-, \text { and } 90- \\
\text { day follow-up as outpatient } \\
\text { Double blind } \\
\text { Medication or PBO given only } \\
\text { during inpatient stay } \\
\text { As needed paracetamol, } \\
\text { nitrazepam, and nicotine } \\
\text { replacement was made available }\end{array}$ & $\begin{array}{l}{ }^{a} \text { Side effects/adverse effects } \\
{ }^{a} C W S \\
{ }^{a} \text { Retention in treatment } \\
\text { Plasma lithium } \\
\text { Plasma oxytocin } \\
\text { Plasma cannabinoids } \\
\text { UCT } \\
\text { SDS } \\
\text { CPQ } \\
\text { SF-12 } \\
\text { DASS-2I } \\
\text { WHOQOL }\end{array}$ \\
\hline $\begin{array}{l}\text { Gabapentin } 1200 \text { mg/ } \\
\text { day (in } 3 \text { divided doses } \\
\text { of } 300 \text { mg QAM, } \\
300 \text { mg Qmidday, } \\
600 \text { mg QHS)+weekly } \\
\text { counseling } \\
\text { Mason et al, } 2012\end{array}$ & $\begin{array}{l}N=50 \text { randomized }(N=25 \\
\text { on gabapentin) } \\
N=18 \text { completers }(N=7 \\
\text { on gabapentin) }\end{array}$ & $\begin{array}{l}\text { Baseline cannabis use: daily users; } \\
\text { used } 11 \pm 18.5 \text { g/week } \\
\text { Age: } 33.9 \pm 9.2 \text { years } \\
\text { Gender }=6 \text { women randomized } \\
\text { Tobacco use: } N=12 \text { randomized } \\
\text { were cigarette smokers }\end{array}$ & $\begin{array}{l}\text { I } 2 \text { weeks; medication titration } \\
\text { complete by day } 4 \\
\text { Medication titrated as tolerated } \\
\text { Double blind } \\
\text { Outpatient setting }\end{array}$ & $\begin{array}{l}{ }^{\mathrm{a} T L F B} \\
{ }^{\mathrm{a} U C T} \\
\text { MWC } \\
\text { BDI } \\
\text { PSQI } \\
\text { MPS } \\
\text { Executive function tasks } \\
\text { Side effects }\end{array}$ \\
\hline $\begin{array}{l}\text { Topiramate } 100 \mathrm{mg} \\
\text { b.i.d.+3 MET sessions } \\
\text { Miranda et al, } 2016\end{array}$ & $\begin{array}{l}N=66 \text { randomized }(N=40 \\
\text { on topiramate) } \\
N=39 \text { completers }(N=19 \\
\text { on topiramate) }\end{array}$ & $\begin{array}{l}\text { Baseline cannabis use: } \mathrm{PBO}, \\
70.94 \% \pm 28.6 \text { of days of } 90 \text {-day } \\
\text { TLFB; topiramate, } \\
70.22 \% \pm 26.89 \text { days of } 90 \text {-day } \\
\text { TLFB } \\
\text { Baseline grams/day: PBO, } \\
0.88 \mathrm{~g} \pm 0.73 \text { per day; topiramate, } \\
0.53 \mathrm{~g} \pm 0.37 \text { per day } \\
\text { Age: PBO, } 18.81 \pm 2.08 \text { years; } \\
\text { topiramate, } 20.30 \pm 2.03 \text { years } \\
\text { Gender: PBO, } 14 \text { women; } \\
\text { topiramate, } 20 \text { women } \\
\text { Cigarettes/nicotine use not } \\
\text { reported }\end{array}$ & $\begin{array}{l}6 \text { weeks, medication titrated over } \\
4 \text { weeks and maintained for } \\
2 \text { weeks before taper } \\
\text { Double blind } \\
\text { Outpatient setting }\end{array}$ & $\begin{array}{l}{ }^{a} T L F B \\
{ }^{a} U C T \\
\text { Side effects } \\
\text { BDI } \\
\text { Neurocognitive testing battery }\end{array}$ \\
\hline $\begin{array}{l}\text { Dronabinol } 20 \text { mg b.i.d. } \\
\text { +weekly counseling of } \\
\text { skills and MET } \\
\text { Levin et al, 201 I }\end{array}$ & $\begin{array}{l}N=156 \text { randomized } \\
(N=79 \text { on dronabinol }) \\
N=99 \text { completers }(N=55 \\
\text { on dronabinol) }\end{array}$ & $\begin{array}{l}\text { Baseline cannabis use: daily } \\
\text { cannabis users ( } 7 \text { and } 30 \text { days); } \$ 5 / \\
\text { day }(\$ 3-\$ 10) ; P B O, 0.5 \mathrm{~g} / \text { day } \\
(0.3-0.9 \mathrm{~g} / \text { day); dronabinol, } 0.6 \mathrm{~g} / \\
\text { day }(0.4-0.9 \mathrm{~g} / \text { day }) \\
\text { Age: } \mathrm{PBO}, 38.4 \pm 9.2 \text { years; } \\
\text { dronabinol, } 36.9 \pm 10.8 \text { years } \\
\text { Gender: } N=28 \text { women } \\
\text { randomized }(\mathrm{N}=12 \text { on } \\
\text { dronabinol) } \\
\text { Cigarettes/nicotine use not } \\
\text { reported }\end{array}$ & $\begin{array}{l}\text { I2 weeks: I-week PBO lead-in; } \\
\text { I-week medication titration; 6- } \\
\text { week maintenance; 2-week } \\
\text { taper; 2-week PBO lead-out } \\
\text { Medication titrated as tolerated } \\
\text { Double blind } \\
\text { Outpatient setting }\end{array}$ & $\begin{array}{l}{ }^{\mathrm{a} T L F B} \\
\text { UCT } \\
\text { MWC } \\
\text { MCQ } \\
\text { Side effects } \\
\text { Study retention }\end{array}$ \\
\hline
\end{tabular}


Table 2 (Continued)

\begin{tabular}{|c|c|c|c|c|}
\hline $\begin{array}{l}\text { Intervention and } \\
\text { publication }\end{array}$ & Number of participants & Participant characteristics & Key study design elements & Outcomes \\
\hline $\begin{array}{l}\text { Dronabinol } 20 \text { mg } \\
\text { +lofexidine } 0.6 \mathrm{mg} \text { ti.d. } \\
\text { +weekly MET/RPT } \\
\text { Levin et al, } 2016\end{array}$ & $\begin{array}{l}N=122 \text { randomized } \\
(N=61 \text { on dronabinol }) \\
N=67 \text { completers }(N=32 \\
\text { on dronabinol })\end{array}$ & $\begin{array}{l}\text { Baseline cannabis use: daily } \\
\text { cannabis users (7 and } 30 \text { days); } \\
\text { PBO, } \$ 20 / \text { day }(\$ 10-\$ 40) ; \\
\text { dronabinol, } \$ 17.6 / \text { day }(\$ 11.5- \\
\$ 24.8) \\
\text { PBO, } 1.6 \mathrm{~g} / \text { day }(0.9-3.8 \mathrm{~g} / \text { day); } \\
\text { dronabinol, } 1.7 \mathrm{~g} / \text { day }(0.8-2.4 \mathrm{~g} / \\
\text { day) } \\
\text { Age: PBO, } 35.4 \pm 10.8 \text { years; } \\
\text { dronabinol, } 34.8 \pm 11.2 \text { years } \\
\text { Gender: } N=38 \text { women } \\
\text { randomized }(\mathrm{N}=22 \text { on } \\
\text { dronabinol) } \\
\text { Cigarettes/nicotine use not } \\
\text { reported }\end{array}$ & $\begin{array}{l}\text { II weeks: I-week PBO lead-in; } \\
\text { I-week medication titration; 6- } \\
\text { week maintenance; 2-week } \\
\text { taper; I-week PBO lead-out } \\
\text { Medication titrated as tolerated } \\
\text { Double blind } \\
\text { Outpatient setting }\end{array}$ & $\begin{array}{l}\text { aTLFB } \\
\text { UCT } \\
\text { MCQ } \\
\text { MWC } \\
\text { Side effects }\end{array}$ \\
\hline $\begin{array}{l}\text { N-acetylcysteine } \\
1200 \text { mg b.i.d.+CM } \\
\text { twice weekly } \\
\text { Gray et al, } 2012\end{array}$ & $\begin{array}{l}N=116 \text { randomized } \\
(N=58 \text { on NAC) } \\
N=106 \text { participants } \\
\text { received at least I dose of } \\
\text { medication (modified ITT) } \\
N=70 \text { completed treatment } \\
(N=37 \text { in NAC) } \\
N=54 \text { participated in } 4- \\
\text { week follow up ( } N=29 \text { on } \\
N A C)\end{array}$ & $\begin{array}{l}\text { Baseline cannabis use: } \\
22.6 \text { days } \pm 7.2 \text { ( } 30 \text { days) } \\
\text { Age: } 18.9 \pm 1.5 \\
\text { Gender: } N=32 \text { randomized } \\
\text { women }(N=19 \text { on NAC) } \\
N=65 \text { cigarette smokers } \\
\text { randomized ( } N=33 \text { on NAC) }\end{array}$ & $\begin{array}{l}8 \text { weeks with 4-week follow-up } \\
\text { visit post medication } \\
\text { Double blind } \\
\text { Outpatient setting }\end{array}$ & $\begin{array}{l}\text { a } U C T \\
{ }^{a} \text { TLFB Side effects }\end{array}$ \\
\hline $\begin{array}{l}\text { Oxytocin } 40 \text { IU before } 2 \\
\text { psychotherapy sessions } \\
+3 \text { sessions of MET } \\
\text { Sherman et al, } 2017\end{array}$ & $\begin{array}{l}\mathrm{N}=16 \text { randomized ( } \mathrm{N}=8 \\
\text { on oxytocin) } \\
\text { I participant in } \mathrm{PBO} \text { group } \\
\text { excluded due to concerns } \\
\text { around data integrity }\end{array}$ & $\begin{array}{l}\text { Unclear baseline cannabis use } \\
\text { Age: } 25.5 \pm 7.6 \text { years } \\
\text { Gender: } 6 \text { women } \\
\text { Cigarettes/nicotine use not } \\
\text { reported }\end{array}$ & $\begin{array}{l}4 \text { weeks; intranasal oxytocin or } \\
\text { PBO administered } 30 \text { min before } \\
\text { the first } 2 \text { MET sessions } \\
\text { Double blind } \\
\text { Outpatient setting }\end{array}$ & ${ }^{\mathrm{a} T L F B}$ \\
\hline
\end{tabular}

Abbreviations: AIS, Athens Insomnia Scale; BIS, Barratt Impulsivity Scale; BTOM-SFS, Brief Treatment Outcome Measure-Social Functioning Scale; CAARS, Conners Adult ADHD Rating Scale-Self; CGI-S and CGI-I, Clinical Global Impression of Severity and Improvement Scales; CIWA, Clinical Institute Withdrawal Assessment; CPQ, Cannabis Problems Questionnaire; CWS, Cannabis Withdrawal Scale; DASS, Depression, Anxiety, and Stress Scale; DTS, Distress Tolerance Scale; HAM-A, Hamilton Anxiety Scale; MCQ, Marijuana Craving Questionnaire; MPS, Marijuana Problems Scale; MWC, Marijuana Withdrawal Checklist; PBO, placebo; PSQI, Pittsburgh Sleep Quality Index; QCQ, Quitting Cannabis Questionnaire; SDS, Severity of Dependence Scale; SF, short form; SMHSQ, St. Mary's Hospital Sleep Questionnaire; STAl, State-Trait Anxiety Inventory; TLFB, Timeline Followback; URICA, University of Rhode Island Change Assessment; WRAADS, Wender-Reimherr Adult Attention

Deficit Disorder Scale; WHOQOL, World Health Organization Quality of Life.

aPrimary outcome measure.

resulting in an underpowered study and conclusions. Further, nefazadone had the greatest proportion of missed doses compared to placebo. The sale of nefazodone was since discontinued in the United States (2003) due to rare but severe hepatotoxicity resulting in fulminant liver failure requiring transplant or resulting in death. Despite identifying these two medications for the purpose of decreasing cannabis use over time by decreasing withdrawal, cannabis withdrawal was not measured specifically, rather only three symptoms of cannabis withdrawal were assessed, two of which can be exacerbated by bupropion (anxiety and irritability), and one in which bupropion is not proposed to improve (sleep). It is 
possible that the high attrition in this study was due to poor tolerance of side effects from the medication, with $45 \%$ of participants on bupropion and $41.7 \%$ of participants on nefazodone reporting side effects. Carpenter (2009), like Haney et al $(2001,2003)$, reached the same conclusion that neither of these medications had sufficient evidence for their clinical utility in the treatment of cannabis withdrawal or CUD. In this instance, the human laboratory data predicted the larger clinical trial results.

Despite these two negative trials (Carpenter et al, 2009; Haney et al, 2001), a subsequent study of bupropion was completed. Penetar et al (2012) utilized the same dose of sustained release bupropion in a randomized, placebocontrolled, double-blind trial between subjects design. The authors utilized a paradigm similar to those used in tobacco cessation trials with a medication lead-in phase (day 8 target quit day). Participants were included if they currently met DSM-IV criteria for cannabis abuse or dependence (which differed from Carpenter et al (2009)) in addition to having at least 3 previous years of heavy use (smoking 5 out of 7 days per week or greater than 25 times per month) plus a past experience of 2 or more negative symptoms of cannabis withdrawal in previous quit attempts. The study was a blend between outpatient clinic setting and human laboratory, requiring time intensive participation on daily visits Monday-Friday for 21 days and weekly MET sessions. Findings included no significant difference between groups in terms of marijuana withdrawal. In the placebo group, post study day 8/'quit day', marijuana withdrawal scores increased significantly in placebo group compared to the bupropion group. Further, the percent change in craving scores were significantly elevated following marijuana cessation in placebo group compared to bupropion group during the withdrawal weeks. Not surprisingly, there were no significant differences between the groups by Actiwatch or sleep diary. The study was notably underpowered and had significant participant attrition. Though the authors surmised that the findings were promising for the possibility of bupropion in the treatment of marijuana withdrawal, this is overstated (Penetar et al, 2012). There are a number of problems with the study, mainly the small number of completers $(N=9)$ on which the findings are based. These individuals that provided weakly positive results were likely highly motivated and committed given the laborious study visits. The authors did not provide reasoning for the high dropout rates, but poor tolerance of the medication due to side effects, as shown in the human lab study (Haney et al, 2001), may be one possibility. Further, the measure of cannabis withdrawal (Marijuana Withdrawal Checklist) used in this study is not a sensitive instrument and has been subsequently shown to not represent cannabis withdrawal (Allsop et al, 2011). While phenotypically, nicotine and cannabis withdrawal appears similar, and sustained release bupropion is effective and FDA-approved for tobacco-smoking cessation, the results of these studies suggest underlying neurobiological differences that do not provide enough evidence to make the medication a viable treatment option for CUD.
The interest in noradrenergic agents has continued in the search for effective CUD treatments. Atomoxetine is a highly selective inhibitor of the presynaptic norepinephrine transporter with low abuse potential. It is FDA-approved for the treatment of ADHD. Because of its action on reuptake inhibition, it increases both synaptic dopamine and norepinephrine mainly in the prefrontal cortex, but not in subcortical areas where there are few noradrenergic nerve terminals (Benn and Robinson, 2017). Tirado et al (2008) studied atomoxetine with flexible dosing (25-80 $\mathrm{mg}$ daily) in an open-label trial in 13 subjects with DSM-IV cannabis dependence to determine feasibility, safety, and tolerability in this population (Tirado et al, 2008). Using a rationale outside of its utility for withdrawal in this open-label study, the authors justified using atomoxetine on observations that cannabis users have symptoms similar to individuals with ADHD, including impairments in attention, executive functioning, and response inhibition. While they found a trend (but not ultimately statistically significant) toward reduction in cannabis use with an increase in percent abstinent days as measured by the Timeline Followback (TLFB) (though not confirmed with collected urinalysis), only 8 subjects completed the study and $\sim 80 \%$ of the subjects experienced significant gastrointestinal side effects of nausea, vomiting, dyspepsia, and loose stools, with two subjects withdrawing due to these side effects. Because of these findings, the authors concluded that atomoxetine did not have a role in the treatment of CUD due to its intolerability in the patient population. Notably, cognitive or behavioral measures of attention, executive functioning, or response inhibition were not measured (Tirado et al, 2008).

Following this open-label trial of atomoxetine for CUD, McRae-Clark et al (2010) published a 12-week outpatient clinical trial of atomoxetine for the treatment of both CUD and ADHD. The authors reasoned that ADHD and CUD highly co-occur together, and as generally accepted in the substance use disorders literature, that treating co-occurring illness frequently improves substance use. In addition to the medication treatment, both groups received MET. While participants on atomoxetine had positive changes in ADHD CGI-Improvement scores, there were no group differences in ADHD symptoms or scores on cannabis use outcomes. Further, participants in the atomoxetine group had notable side effects with all participants on active medication reporting some side effects and with gastrointestinal side effects being 2.25 times higher as compared to placebo. Of note, only $15 \%$ of the initially randomized participants completed the trial. The authors did not fully elucidate the reasons for high attrition in the study, but as demonstrated in the open-label trial, atomoxetine produces significant side effects in cannabis users (McRae-Clark et al, 2010).

Additional evidence demonstrates the potentially adverse outcomes associated with the use noradrenergic agents in the treatment of CUD. Levin et al (2013) looked at extended release venlafaxine in the outpatient treatment of cannabis dependence and co-occurring major depressive disorder or dysthymia. Venlafaxine-extended release was selected 
because of its dual action on serotonin and norepinephrine reuptake inhibition, hypothesizing that improved mood would reduce cannabis use. Primary outcome measures, including abstinence from cannabis as confirmed by urine toxicology and self-report for 2 consecutive weeks in addition to the Hamilton-Depression (HAM-D). Results demonstrated that both groups had improvement in mood; however, abstinence rates were low and worse on venlafaxine-extended release $(11.8 \%$ on active medication vs $36.5 \%$ on placebo) (Levin et al, 2013). A secondary analysis (Kelly et al, 2014) demonstrated that the venlafaxine group had more severe withdrawal symptoms, despite not changing their cannabis use. The authors concluded that the worse 'withdrawal symptoms' were likely secondary to commonly occurring side effects of venlafaxine that may have contributed to continued cannabis use.

A final published study from the human laboratory in a model of relapse prevention, examined mirtazapine. Mirtazapine is an antidepressant that enhances noradrenergic and serotonergic transmission by blocking presynaptic inhibitory alpha 2 autoreceptor, resulting in sedation and increased appetite. Many cannabis users identify the sedative and appetitive benefits of cannabis. Insomnia and decreased appetite are problematic symptoms in withdrawal. Haney et al (2010) investigated mirtazapine as a candidate medication in the treatment of CUD. The authors demonstrated that mirtazapine improved food intake and sleep during withdrawal; however, it had no effects on preventing relapse and no impact on other symptoms of cannabis withdrawal, including mood. Though potentially useful in targeting insomnia and food intake during cannabis withdrawal, mirtazapine monotherapy is likely not sufficient as treatment for CUD (Haney et al, 2010).

Data from the literature on noradrenergic agents suggest that non-stimulant cognitive and mood-enhancing medications are not promising compounds for the treatment of CUD. At best, they may be used to target some specific symptoms of cannabis withdrawal and at worst, they may cause intolerable side effects, particularly gastrointestinal, and/or exacerbate cannabis use.

\section{Serotonergic Agents}

Building off of the idea of treating specific symptoms of cannabis withdrawal, interest developed in agents with low abuse potential to treat anxiety. Anxiety was thought to be a major factor in the continued use of cannabis. Previous studies had demonstrated that anxiety correlated with increased cannabis withdrawal (Budney et al, 1999) and was associated in the use of cannabis to cope (Buckner et al, 2008). Further, it was shown that reductions in anxiety led to reductions in cannabis use (Buckner and Carroll, 2010). Buspirone, the serotonin $1 \mathrm{~A}$ partial agonist used clinically in the treatment of anxiety and augmentation of antidepressants for depressive disorders, was selected as a candidate agent in the treatment of CUD because of preclinical evidence that the serotonin system had a significant role and interaction with cannabinoids (Hill et al, 2006; Gomes et al, 2011; Zanelati et al, 2010). It was hypothesized that buspirone's anxiolytic effects may be helpful in preventing a relapse to cannabis use (McRae-Clark et al, 2009).

Following an open-label trial demonstrating tolerability and a pilot controlled trial that demonstrated buspirone led to a reduction in the percentage of positive urine cannabinoid tests among treatment completers (McRae-Clark et al, 2009), this same research group completed a fully powered randomized controlled trial of buspirone for the treatment of CUD (McRae-Clark et al, 2015). This larger study also looked to explore the impact of serotonin allele variations on buspirone treatment response. Interestingly, this study did not assess withdrawal symptoms, despite buspirone's anxiolytic activity and the notable symptom of anxiety in cannabis withdrawal. While $90 \%$ of the sample received at least one dose of medication, less than half of the participants completed the trial. There was no effect of treatment on cannabis use outcomes with overall low abstinence rates. Craving decreased across both groups over time, and the serotonin 1A receptor genotype had no relationship with outcomes. The authors identified that the included participants in the study had low anxiety as measured with the HAM-A and exclusion criteria eliminated individuals with significant psychiatric disorders or who were taking other psychoactive substances. The authors suggested that buspirone may be helpful for individuals with CUD and cooccurring anxiety disorders, though this is speculative. An important finding that likely has clinical treatment implications was that women had a greater likelihood of having a negative urine cannabinoid test if they were on placebo $(12.9 \%)$ as compared to buspirone $(2.4 \%)$, while men were more likely to have a negative urine on buspirone $(8.7 \%$ compared to $4.5 \%$ ). There were fewer women in the previous pilot controlled trial (McRae-Clark et al, 2009) as compared to this study, which was one identified factor the authors reasoned may have contributed to the difference in outcomes across the two trials (McRae-Clark et al, 2015). This finding suggests that gender appears to be a critical independent variable in the development and evaluation of new treatments for CUD, though few studies are published examining its role in treatment outcomes.

Weinstein et al (2014) also looked at the selective serotonin reuptake inhibitor, escitalopram in combination with CBT, and its effects on abstinence rates in CUD. The authors found that the medication provided no benefit over placebo. Overall, the study demonstrated a low rate of abstinence, with no significant difference between active medication and placebo, and once again, high participant attrition (Weinstein et al, 2014).

Despite these negative studies of a selective serotonin reuptake inhibitor and serotonin $1 \mathrm{~A}$ active agent, in conjunction with one additional negative study of the use of fluoxetine (selective serotonin reuptake inhibitor) in the treatment of CUD and co-occurring depressive disorders in adolescents (Cornelius et al, 2010), McRae-Clark et al (2016) 
explored vilazodone in the treatment of CUD. Vilazodone has serotonin $1 \mathrm{~A}$ agonism in combination with selective serotonin receptor inhibition. The authors reasoned that vilazodone's dual action would more effectively target the withdrawal symptoms of anxiety and depression, yet similarly to the gap in the buspirone trial, cannabis withdrawal symptoms were not assessed as an outcome. The authors found that while both groups reduced their use during the study, there was no benefit to being on vilazodone, and overall abstinence rates were low. Though the sample was underpowered and had high attrition, gender differences were explored and found a nonsignificant trend that men treated with vilazodone had an increased likelihood of lower urine cannabinoid levels than women on active treatment, suggesting important gender implications in treatment choice (McRae-Clark et al, 2016), as was previously found with buspirone. The population had low baseline levels of anxiety and depression so changes could not be assessed, though the authors concluded that anxiety and depression may not be clinically relevant targets in medication treatment for CUD.

While there are inconsistencies in the rationale driving the selection of treatment agent with outcome measures assessed, the studies investigating serotonin $1 \mathrm{~A}$ active agents do contribute a helpful clinical perspective in terms of elucidating a key and influential clinical characteristic on treatment selection and thereby outcomes for CUD-gender. A human laboratory study evaluating for sex differences found that women may be more sensitive to the subjective effects related to the abuse liability of cannabis relative to men, which could potentially lead to greater challenges in finding successful treatments (Cooper and Haney, 2016). This may be a factor in the findings by McRae-Clark et al $(2015,2016)$ that women did worse than men with regards to their clinical response. In most of the published studies evaluating novel agents for the treatment of CUD, men make up the majority of the participants leading to statistical barriers in exploring the role of gender. However, when powered to do so, gender may be playing an important role in the findings and should be explored.

The work to date exploring antidepressants and atypical anxiolytics appear to have limited value in the treatment of CUD other than for the potential treatment of comorbid conditions or targeting specific symptoms that trouble patients (ie, food intake and sleep). Further, medication selection likely depends on specific patient characteristics, such as gender.

\section{Gama Aminobutyric Acid Agents}

Targeting specific symptoms of depression or anxiety did not prove fruitful in the treatment of CUD. Following the reasoning that cannabis withdrawal results in a broader range of symptoms, including irritability, mood lability, temper outbursts, anxiety, insomnia, and nausea, research looked to utilizing medications that mitigate these symptoms. The next phase of studies explored the use of classic mood stabilizers and antiepileptic drugs.
In 2004, two studies were published looking at divalproex in the treatment of CUD: one in the human laboratory setting (Haney et al, 2004); and one pilot-controlled trial in an outpatient clinic setting (Levin et al, 2004). Haney et al (2004) found that while divalproex decreased craving during abstinence it also increased ratings of being anxious, irritable, tired, 'bad effect', worsened mood, and cognitive performance. Levin et al (2004) conducted the first trial in a clinic setting exploring pharmacological treatment for CUD using divalproex. As this was the first outpatient medication treatment trial for the CUD, the primary aims differed from future studies. The authors aimed to evaluate (1) whether cannabis-dependent participants would seek pharmacological treatment, (2) whether they can be maintained in an outpatient medication treatment trial, and finally (3) whether participants reduced self-report of frequency and amount of cannabis use in addition to irritability on divalproex sodium (titrated based on blood levels between 50 and $120 \mu \mathrm{g} / \mathrm{ml}$ ). Findings concluded that cannabis-dependent individuals were interested in seeking outpatient medication treatment and could engage throughout the trial. As predicted by the human lab findings, while both groups reduced cannabis use and had reductions in irritability, divalproex had no added benefit. Further, it appeared participants were poorly adherent to the medication as reflected in low blood levels of divalproex sodium. Both the human laboratory and outpatient clinical treatment trial concluded that divalproex sodium has no role in the treatment of CUD.

In line with trying to reduce cannabis withdrawal symptoms, other medications with gamma aminobutyric acid (GABA) receptor activity have been studied as potential treatments for CUD with some promising findings. Haney et al (2010) investigated baclofen. Bacolfen is a GABA-B receptor agonist approved for the treatment of spasticity in neurological disorders like multiple sclerosis. The authors reasoned that the medication's sedating properties may help to alleviate cannabis withdrawal. Further the authors cited evidence from previous studies of baclofen's ability to reduce self-administration (Haney et al, 2006) and improve mood symptoms (Addolorato et al, 2000) in the context of other drugs of abuse (cocaine and heroin) as further support for its role in treating CUD. While Haney et al (2010) found baclofen dose dependently reduced craving for cannabis, baclofen did not reduce relapse nor did it have an effect on mood. Contradictory to the hypothesized effect, baclofen worsened one measure of sleep and cognitive performance, leading the authors to conclude that its overall utility in the treatment of cannabis withdrawal or CUD was limited (Haney et al, 2010).

Investigation of the GABA-A agonists has proven more promising. Vandrey et al (2011) looked at extended release zolpidem specifically for treating sleep difficulty in the context of cannabis withdrawal in a human laboratory model of abstinence. The authors reasoned that sleep difficulty is consistently rated as one of the most severe symptoms in cannabis withdrawal (Vandrey et al, 2008). The authors also highlighted how this may negatively impact attempts to stop 
cannabis use given previous findings that sleep difficulty frequently contributed to relapse during times of abstinence on quit attempts (Hughes et al, 2008). Using the gold standard of polysomnography (PSG) for objective sleep measures, in conjunction with subjective ratings and nextday cognitive performance measures, Vandrey et al (2011) found that extended release zolpidem attenuated the effects of abstinence on sleep architecture as measured with PSG in addition to improvements in subjective ratings of sleep quality. There were no significant side effects or negative impact on next-day cognitive performance (Vandrey et al, 2011). The authors concluded that it may be useful as an adjunctive medication treatment for CUD. Though not acknowledged in the paper, zolpidem is a benzodiazepinereceptor-like drug and has been found to have greater abuse potential than other sleep agents, dangerous withdrawal symptoms when dependence is established, and problematic side effects, including parasomnias in the context of amnesia (Keuroghlian et al, 2012). The potential for misuse and negative health effects should be considered if zolpidem is used to target insomnia in patients with CUD. Consistent with the above findings, a secondary analysis of a study looking at lithium carbonate for CUD (Johnston et al, 2014) found that individuals who received nitrazepam as needed for insomnia had improved objective measures of sleep as recorded with actigraphy, though subjective ratings of sleep did not change (Allsop et al, 2015).

Gabapentin is a medication that indirectly modulates GABAergic mechanisms through its blockage of the alpha $2 \mathrm{~d}$ subunit of voltage-gated calcium channels at selective presynaptic sites. Preclinical evidence demonstrated that gabapentin modulates an anxiogenic-like state as a result of increased extrahypothalamic corticotrophin-releasing factor in cannabis withdrawal states, restoring brain homeostasis in the context of stress (Roberto et al, 2008). Further, clinical evidence showed gabapentin reduces craving and disturbances in sleep and mood in alcohol withdrawal and enhanced cognitive performance (Ghaemi et al, 1998; Lo et al, 2010; Mason et al, 2009). A pilot-controlled trial by Mason et al (2012) investigated the role of gabapentin in the treatment of CUD. Gabapentin demonstrated significant reductions in urine cannabinoids, self-report of cannabis use, withdrawal symptoms including craving, and problems secondary to marijuana use. Gabapentin also improved executive functioning in the study, though it could not be determined whether it was a direct effect of gabapentin or a secondary effect as a result of improved withdrawal and decreased cannabis use. Though medication adherence was high, study completion was poor as with previous trials, with only a $36 \%$ completion rate. The authors identified younger age of first cannabis use, years of daily cannabis use, cannabis withdrawal severity, impaired ability to inhibit impulses, and difficulty processing complex information as predictors of dropout. Targeting these factors that have malleability may improve outcomes across CUD treatment trials. Though a relatively small placebo-controlled pilot, overall findings from this study provided encouraging evidence for the use of gabapentin in the treatment of CUD (Mason et al, 2012). A larger, fully powered controlled trial is pending results and will provide more conclusive evidence for the role of gabapentin in treating CUD (NCT0094376).

A final study of GABAergic medications by Miranda et al (2016) looked at topiramate in adolescents and young adults in the treatment of CUD. Topiramate blocks voltagesensitive sodium and calcium channels that results in the potentiation of GABA with enhancement of GABA-A receptor function in addition to antagonist activity at AMPA/kinate glutamate receptors. This activity was reasoned to be helpful in potentially decreasing the acute reinforcing effects of cannabis. In a placebo-controlled pilot of youth with CUD, the authors found that while abstinence rates were not different between the groups, participants receiving topiramate reduced their use significantly as measured in grams per day. However, the youth poorly tolerated the topiramate and this arm had higher dropout rates compared to the placebo group, with participants identifying side effects as the primary reason for leaving the study. While the authors concluded that the poor tolerance of the medication and lack of efficacy on abstinence rates likely suggest limited utility in the treatment of CUD, we find the topiramate's effects on reducing grams of cannabis used per day encouraging (Miranda et al, 2016). Slower titration and lower doses of medication in addition to a good working treatment alliance with a patient with close follow-up may mitigate the risk of patient dropout from treatment to achieve reductions in cannabis use. Further, adults may tolerate the medication differently than youth. Topiramate has demonstrated clinical utility in the treatment of other substance use disorders in adult populations, including nicotine and alcohol (Anthenelli et al, 2017).

GABA-A agonist sleep agents and other medications with GABA-A activity, such as gabapentin and topiramate, show promise in the treatment of CUD to target difficulties with sleep as a result of withdrawal and/or maintenance treatment of CUD by decreasing cannabis use, respectively. Larger, fully powered placebo-controlled trials need to be completed.

\section{CANNABINOID APPROACHES}

\section{CB-1 Receptor Agonists}

Agonist approaches have been considered in the treatment of CUD given the success of conceptually similar treatments for nicotine and opioid use disorders. Agonist medication not only has the benefit of suppressing withdrawal, but also may attenuate the acute effects of drug use. The former is key in preventing relapse during quit attempts, and the latter in initiating and maintaining abstinence or reduced use. Generally, an ideal agonist substitute in the treatment of substance abuse has the following properties: low abuse potential; less hazardous route of administration; functions to reduce withdrawal symptoms and craving; decreases the reinforcing effects of the target drug, in this case, cannabis 
and its most psychoactive component THC, and leads to an improvement in functioning (Balter et al, 2014).

A number of human laboratory studies have looked at dronabinol, oral THC, which has FDA indications for the treatment of anorexia associated with AIDS and second-line treatment for nausea and vomiting associated with cancer chemotherapy in doses up to $20 \mathrm{mg} /$ day. Hart et al (2002) evaluated the dose effects of dronabinol on subjective effects of smoked cannabis. This study found that $80 \mathrm{mg}$, but not $40 \mathrm{mg}$, attenuated the subjective effects of smoked cannabis, noting a 50\% reduction in 'good effect.' Haney et al (2004, 2008) was the first to look at oral THC and its effects on cannabis withdrawal and a model of relapse, finding that dronabinol decreased certain withdrawal symptoms including craving, decreased food intake, physical symptoms, and mood disturbance, without producing intoxication, but failed to prevent relapse as compared to placebo (Haney et al, 2004; Haney et al, 2008). Budney et al (2007) looked at two doses of dronabinol in an outpatient human laboratory study of non-treatment-seeking heavy cannabis users. The authors found that while both doses reduced withdrawal symptoms, the higher dosage produced additional suppression of symptoms such that ratings returned to baseline when participants were smoking as usual (ie, limited to no withdrawal symptoms) (Budney et al, 2007). Vandrey et al (2013) examined dose effects of dronabinol. The authors found that dronabinol dose-dependently decreased withdrawal symptoms with few adverse effects or problems with cognitive performance (Vandrey et al, 2013). Surprisingly, he did not demonstrate any alteration in subjective effects of smoked cannabis on any dose, as was previously found in Hart et al (2002), though attenuation in increases in heart rate were seen. In summary, the human lab studies of oral THC have some mixed results regarding its impact on subjective effects of smoked cannabis in addition to failing to prevent relapse, though provided strong evidence for the role of oral THC (dronabinol) in dose-dependently attenuating withdrawal at higher than FDA-approved doses in nontreatment-seeking, heavy cannabis users.

Providing further proof of concept that the human lab can serve as a predictive model for clinical trials, Levin et al (2011) studied dronabinol in a fully powered placebocontrolled trial for the treatment of CUD. Primary cannabis outcomes included the TLFB. While urine was collected for cannabinoid testing in the placebo group to correlate selfreport, urine results in the dronabinol group were not used due to the medication's confounding effects on urine cannabinoid testing. Levin et al (2011) found no effect of dronabinol on abstinence compared to placebo, though withdrawal symptoms were significantly lower and study retention was greater ( $77 \%$ vs $61 \%)$ on dronabinol compared to placebo. Notably, pre-treatment withdrawal symptoms were not assessed to provide a baseline measure to compare to results during treatment. The comparison of dronabinol to placebo with regards to withdrawal also included all study participants and not just those who reduced or abstained from cannabis, suggesting that changes in 'withdrawal' may be encompassing other factors as well. As a result, it is difficult to assess the magnitude with which dronabinol suppressed withdrawal; however, given the limitations, the findings are consistent with the human lab findings.

Moving toward combination strategies, Haney et al (2008), looked at oral THC in conjunction with lofexidine for its effects on cannabis withdrawal, craving, and relapse in the human lab. Lofexidine was selected because of its reported favorable side-effect profile compared to other alpha 2 adrenergic agonists (eg, clonidine and guanfacine) in conjunction with preclinical data demonstrating that noradrenergic hyperactivity contributes to withdrawal from cannabinoids (Hart et al, 2005). The study found that THC alone again decreased a subset of withdrawal symptoms, but failed to decrease relapse. Lofexidine alone was found to have side effects of sedation and worse abstinence-related anorexia but did improve sleep and decrease relapse to smoking cannabis. As hypothesized, the combination provided the most robust improvements in sleep, reductions in cannabis withdrawal, craving, and relapse with $50 \%$ of the participants choosing not to purchase puffs of cannabis for the duration of the relapse phase (Haney et al, 2008).

Following the robust results of this human lab study, Levin et al (2016) took the combination of dronabinol and lofexidine to the clinic setting. This time, however, there was a discrepancy between the lab and clinic setting findings. There was no difference between active medication and placebo with regards to self-reported rates of abstinence, and both groups showed reductions in cannabis use over time with half of all patients reporting a reduction in use of $50 \%$ or more (Levin et al, 2016). There was also no difference in withdrawal scores over time. There was an overall low percentage of participants completing the medication phase, particularly in the active medication arm, and lower doses were required given intolerable side effects, including dry mouth, intoxication, and hypotension. Surprisingly, the combination treatment was not more effective than placebo for promoting abstinence, reducing withdrawal symptoms, or retaining individuals in treatment. Some key differences between the lab study and the clinic setting included (1) tolerance to the medication and dosing, (2) duration of medication treatment, (3) inpatient $v s$ outpatient setting, and (4) relapse prevention $v s$ abstinence initiation design, all of which likely factored into the differences in the studies' outcomes. Future studies should factor in these considerations, particularly when translating human laboratory findings to the clinical setting.

The mostly negative results of dronabinol in the treatment of CUD likely has to do with its poor bioavailability (Bedi et al, 2013), in conjunction with the differences in study designs. Its slow onset and long duration of action can decrease craving and symptoms of withdrawal at doses that should produce minimal intoxication and thereby give it a role in this specific component of treatment. However, its mixed effects on attenuating subjective effects and inability to impact reductions or abstinence rates in the clinic setting suggest it is not sufficient as a monotherapy or in combination with lofexidine in the treatment of CUD. 
Given the issues with dronabinol, research has pursued other agonist formulations with better medication profiles as potential treatments for CUD. Nabilone, a DEA Schedule II (dronabinol is Schedule III) potent synthetic cannabinoid is currently FDA-approved up to $6 \mathrm{mg} /$ day for the second-line treatment of nausea and vomiting related to cancer chemotherapy treatment. It has better oral bioavailability, improved efficacy, and a more linear dose effect than dronabinol (Bedi et al, 2013). Because it is a synthetic THC analog, it has distinct urinary metabolites that do not crossreact with THC metabolite assays allowing for monitoring with urine cannabinoid testing as usual (unlike dronabinol, which is synthetic THC). To date, two human laboratory studies using nabilone, one as a monotherapy and one in combination with zolpidem, have demonstrated encouraging results for its role in the treatment of CUD.

Haney et al (2013b) looked at dose effects of nabilone and compared it to placebo in the human lab setting to assess its impact on cannabis withdrawal and relapse (Haney et al, 2013b). The study found that both doses of nabilone significantly decreased cannabis self-administration as a model of relapse, in addition to reducing ratings of irritability and 'bad effect' during precipitated abstinence. High-dose nabilone $(8 \mathrm{mg})$ also decreased craving. Nabilone reversed abstinence-induced sleep disturbances and changes to food intake. Placebo was associated with better performance on the cognitive tasks during abstinence. This study demonstrated that nabilone significantly reversed characteristic and problematic symptoms of cannabis withdrawal in addition to decreasing a model of relapse, by not only decreasing cannabis self-administration but reducing the use of cannabis in those who had relapsed from their baseline use. While there is a theoretical risk of abuse that is greater with nabilone than oral THC, at least in this study, participants reported few subjective effects from nabilone. It is likely that nabilone's properties as a long-acting and slow onset of action agonist may make it less likely to have the abuse liability of smoked cannabis. Overall, nabilone shows promise as a medication treatment in the prevention of relapse, particularly using the lens of harm reduction. The authors concluded that further research of nabilone for the treatment of CUD is needed in the clinic setting and in the context of abstinence initiation. Interestingly, a subsequent secondary analysis of this study in addition to four other lab studies (lofexidine, dronabinol, mirtazapine, and quetiapine) found that individuals who smoked tobacco cigarettes had a much greater likelihood of relapse to cannabis than nontobacco smokers (Haney et al, 2013a), identifying a key factor that is rarely looked at in CUD treatment trials. Cigarette smoking may be a marker for greater severity of CUD and/or it may be contributing to the limited effects of medications seen in treatment trials and should be assessed in future studies.

Given the positive lab findings of nabilone and zolpidem as monotherapies, a combination of the two medications was evaluated in the human lab as compared to zolpidem monotherapy (Herrmann et al, 2016). In this study, heavy cannabis users were evaluated for their experience of cannabis withdrawal symptoms and relapse. The study found that while both medication exposures decreased withdrawal-related sleep disturbances, only the combination attenuated withdrawal-related mood and food changes in addition to decreasing self-administration of active cannabis. While neither medication treatment changed cognitive performance, the combination medication did produce modest increases in abuse-related subjective ratings of capsules. It can be surmised from this second lab study that nabilone drove the decreases in self-administration and should be evaluated in clinical treatment trials for CUD. One limitation of the study was the fourth treatment arm of nabilone monotherapy was not included for comparison.

A final agonist formulation of nabixmols has been investigated for its role in the treatment of cannabis withdrawal and CUD. Nabixmols contains extracts from the Cannabis sativa plant. These include THC, which would provide the agonist action, and cannabidiol (CBD), a cannabinoid with proposed effects on attenuating paranoia, euphoria, anxiety, and depression (Vermersch, 2011). They are in a 1:1 ratio that is administered through buccal spray that provides a more rapid onset of action and more favorable pharmacokinetics as compared to oral THC (Vermersch, 2011). Allsop et al (2014) looked at nabixmols during an inpatient admission followed by a 28 -day period without medication. The study found that nabixmols significantly reduced the overall severity of cannabis withdrawal compared to placebo during the inpatient admission, including reductions in symptoms of irritability, depression, and craving, with limited but positive improvements in sleep, anxiety, appetite, physical symptoms, and restlessness. Nabixmols further reduced the time course of withdrawal symptoms during the inpatient admission by almost 2 days (Allsop et al, 2014). Patients receiving nabixmols stayed in inpatient treatment longer, reported no greater intoxication, could not distinguish it from placebo spray, and had no difference in the number or type of adverse events. Though there were no differences in time to relapse after discharge from the inpatient setting, both groups reported reduced use of cannabis as confirmed with urine cannabinoid testing at follow-up, suggesting that inpatient treatment may be a stimulus for reduced use. Alternatively, inpatient treatment for CUD may also select for the most change-oriented and motivated patients. Given the positive impact on withdrawal but the lack of maintenance nabixmols treatment, a followup trial is now underway. It is not surprising that acute detoxification without maintenance medication treatment did not lead to differences in abstinence, reductions in cannabis use, or time to relapse following treatment, as this is consistent with other acute substance abuse treatment episodes without continued care. A recent open-label outpatient case series demonstrated good tolerability of nabixmols in addition to reductions in cannabis use, craving, and withdrawal, warranting further exploration of nabixmols in the treatment of CUD (Trigo et al, 2016a; Trigo et al, 2016b). As the added benefit of CBD is debatable, future 
trials should also do a direct comparison of nabixmols to another agonist, such as nabilone or oral THC, and if possible, by same route of administration (buccal).

\section{What About CBD Alone?}

It has been proposed that $\mathrm{CBD}$ may attenuate the euphoric effects of smoked THC and reduce intoxication (Morales et al, 2017); thus, it has been investigated as a potential treatment for CUD. CBD has a varied and complex mechanism of action, with no direct effects at $\mathrm{CB}-1$ receptors. Given its potential therapeutic benefit, Haney et al (2016) completed a human lab study to assess the influence of a range of single doses of $\mathrm{CBD}$ on the reinforcing, subjective, cognitive, and physiological effects of smoked cannabis as compared to placebo during eight outpatient sessions. In this systematic study, the authors found that acute oral CBD pre-treatment did not alter subjective, reinforcing, or cardiovascular effects of smoked cannabis in heavy cannabis users (Haney et al, 2016). While they did not study chronic administration nor the same route of administration as the nabixmols study (oral $v s$ buccal), both of which may have important implications for the impact of medication treatment (Haney and Spealman, 2008), the investigators concluded that oral CBD likely does not have a role as a potential medication treatment for CUD. The authors' findings (Haney et al, 2016) suggest that the results from the Allsop et al (2014) study may have been driven by the THC in nabixmols.

\section{CB-1 Receptor Antagonists}

The use of antagonist approaches by directly decreasing the subjective and reinforcing effects of a drug of abuse has demonstrated success in the treatment of other substance use disorders. Rimonabant is a high-affinity CB-1 receptor antagonist. Human lab studies of both a single high dose (90 mg) of rimonabant (Huestis et al, 2001), and repeated lower doses $(40 \mathrm{mg}$ ) demonstrated reduced physiological and subjective effects of smoked cannabis (Huestis et al, 2007). However, the serious adverse psychiatric effects of rimonabant, including anxiety, depression, and suicidality leading to the discontinuation of its use in clinical trials preclude further investigation or feasibility of its role in the treatment of CUD (Roberfroid et al, 2010). It is speculated that the rimonbant's inverse agonist properties may be responsible for these adverse effects and that the development of a neutral antagonist may be a promising treatment in CUD (Balter et al, 2014). Preclinical studies are currently evaluating these potential agents (Gueye et al, 2016), and those with promising preclinical findings and evidence of safety should be investigated in future clinical research.

In summary, medications with $\mathrm{CB}-1$ receptor agonism are effective in treating cannabis withdrawal. $\mathrm{CB}$ receptor agonists' role in the overall treatment of CUD is yet to be determined. Promising human laboratory work with nabilone and recent evidence of nabixmols in the inpatient treatment setting warrant further investigation into their usage in initiating abstinence or reductions in cannabis use in the outpatient treatment setting in addition to how maintenance treatment following inpatient detoxification may further prevent relapse. Oral CBD does not appear to have a role in treating CUD, though further investigation with regards to the effects of route of administration and combination with THC should be pursued. CB-1 receptor antagonists with a more favorable side-effect profile are in preclinical testing stages (Gueye et al, 2016), and promising agents may have a role in the treatment of CUD.

\section{OTHER AGENTS?}

\section{Naltrexone}

Other agents have been considered in the reduction of positive and subjective reinforcing effects of cannabis, including the mu opioid receptor antagonist, naltrexone. While one human lab study demonstrated that acute treatments with different doses of naltrexone before smoking cannabis increased the positive subjective effects of cannabis in non-treatmentseeking heavy cannabis users (Cooper and Haney, 2010), a second placebo-controlled human laboratory study with chronic naltrexone administration demonstrated a significant reduction in both active cannabis self-administration and selfreport of positive effects as compared to placebo (Haney et al, 2015). The effects continued outside the lab. Maintenance administration of naltrexone more accurately represents the use of this medication in the clinical setting. Given that chronic administration of naltrexone has been shown to reduce self-administration in the lab, further investigation of this medication in outpatient treatment settings is warranted.

\section{$N$-acetylcysteine}

$\mathrm{N}$-acetylcysteine (NAC) is a widely available, over-thecounter supplement. NAC is a prodrug of the amino-acid cysteine. Cysteine has an important role as a main component in the cysteine-glutamate exchanger, which controls glutamate levels. Because of increasing interest in the role of glutamate in substance use, NAC was investigated as a candidate pharmacotherapy for CUD. Following an encouraging open-label pilot trial (Gray et al, 2010), Gray et al (2012) completed a fully powered randomized controlled trial of NAC for the treatment of CUD in adolescents and young adults. Participants in the NAC group had more than twice the odds of having a negative urine cannabinoid test compared to placebo. NAC was well tolerated with minimal adverse events. A secondary analysis of this study identified important characteristics of the participants that resulted in a differential treatment effect. Low baseline impulsivity, high medication adherence, and low baseline severity of cannabis use as measured by urine cannabinoid levels predicted a better treatment outcome with higher likelihood of negative urine testing (Bentzley et al, 2016). Future research should consider assessing these factors to better understand the effects of medications. 
Identifying these subgroups of participants that may benefit from the mitigation or enhancement of these malleable factors (such as impulsivity or medication adherence) will likely lead to improvements in CUD treatment. This study was recently replicated in adults (Gray et al, 2017), but the overall results of the study were negative, with NAC showing no demonstrative benefit as compared to placebo. However, post hoc analyses demonstrated that the younger adults had better outcomes, suggesting a possible age effect on treatment (Gray et al, 2017).

\section{Lithium Carbonate}

Following the same reasoning behind the selection of the GABAergic agents, lithium carbonate was studied for its potential impact on mood stabilization with the added benefit of a reduction in depression during cannabis withdrawal (Johnston et al, 2014). Preclinical evidence demonstrated lithium-reduced symptoms of cannabis withdrawal by stimulating release of oxytocin (Cui et al, 2001). Clinical evidence from an open-label pilot showed lithiumameliorated cannabis withdrawal symptoms (Winstock et al, 2009). There was also speculation that lithium may help eliminate THC through its actions on metabolism and excretion (Johnston et al, 2014). For these reasons, Johnston et al (2014) explored the use of lithium carbonate in adults with CUD who were admitted to an inpatient unit for detoxification from cannabis. Unlike the previous treatment studies in the outpatient setting and similarly to human laboratory studies, the controlled inpatient environment induced cannabis withdrawal with known cessation of cannabis use. Withdrawal symptoms were measured with greater confidence in their underlying etiology, as compared to ambiguity in the outpatient setting as a result of less controlled factors. Results demonstrated no significant impact of lithium over placebo on withdrawal symptoms in the inpatient setting in addition to no differences in inpatient retention, adverse events, plasma oxytocin, or cannabinoid levels. While the study was underpowered to fully detect a difference, the authors still concluded that lithium carbonate likely has no role in the treatment of acute cannabis withdrawal symptoms (Johnston et al, 2014).

\section{Quetiapine}

Quetiapine is an atypical antipsychotic with a complex mechanism of action involving serotonin $2 \mathrm{~A}$, dopamine 2, histamine 1, adrenergic alpha 1 and 2 receptor antagonism, serotonin 1 A partial agonism, and inhibition of the norepinerphrine transporter. Because of these multiple sites of action, quetiapine is a multifunctional medication with effects on reducing insomnia, stabilizing mood, and improving appetite. Cooper et al (2013) studied the effects of quetiapine $200 \mathrm{mg}$ as compared to placebo on cannabis withdrawal and relapse in the human laboratory. Compared to placebo, this dose of quetiapine improved sleep quality, increased caloric intake, and decreased weight loss during withdrawal. However, quetiapine paradoxically increased cannabis craving and self-administration of cannabis during the relapse phase. On the basis of the findings, the authors concluded that while quetiapine successfully attenuated key symptoms in cannabis withdrawal, it did not demonstrate an ability to reduce relapse to cannabis and may even exacerbate craving, potentially driving cannabis use (Cooper et al, 2013).

An open-label pilot of chronic dosing of quetiapine (600 mg max daily dose) resulted in reduced dollar amount of cannabis used as confirmed with a reduction in urine cannabinoid levels by almost 50\% (Mariani et al, 2014). Participants had side effects of fatigue and somnolence, and reduced dosing was needed with an identified target dose of $300 \mathrm{mg}$ nightly (Mariani et al, 2014). The study did not assess craving nor withdrawal, although these were proposed targets for the rationale in selecting quetiapine. These promising findings in this open-label trial served as the basis for a larger placebo-controlled trial investigating quetiapine for the treatment of CUD in the outpatient setting that recently completed enrollment, with completion of the trial and results pending (NCT01697709). Other atypical antipsychotics, including olanzapine and clozapine, have been explored for their impact on CUD in the context of co-occurring chronic psychotic disorders with mixed though mostly negative results. This topic is reviewed in more detail in a previous paper (Gorelick, 2016).

\section{Oxytocin}

A recent small placebo-controlled pilot by Sherman et al (2017) explored the role of oxytocin in enhancing psychosocial treatment for CUD. Oxytocin is a hypothalamic neuropeptide that has a critical role in social cognition and behavior. Previous studies have demonstrated that oxytocin reduces craving for cannabis in humans (McRae-Clark et al, 2013), and endocannabinoid signaling appears to mediate oxytocin social reward (Wei et al, 2015). Individuals with CUD received either oxytocin or placebo intranasally before three sessions of MET for the treatment of CUD. There was no overall treatment effect of oxytocin on mean daily cannabis use amount between sessions; however, there was a significant decrease in cannabis use on the day oxytocin and MET was administered as compared to placebo. The authors concluded that oxytocin may enhance the effects of MET on cannabis use outcomes. A limitation of the pilot was the lack of assessments for confirmatory cannabis biomarkers (urine cannabinoid testing) and prosocial behavior (the proposed mechanism of action).

\section{CONCLUSIONS AND FUTURE RESEARCH DIRECTIONS}

\section{Summary}

On the basis of the current available literature of placebocontrolled trials for CUD (Table 3 for summary), there is laboratory evidence suggesting that mirtazapine and 
quetiapine reduce some symptoms of cannabis withdrawal, though these medications do not effect cannabis use. Zolpidem and possibly benzodiazepines, like nitrazepam, may be useful in targeting sleep disturbances as a result of cannabis withdrawal. Oral THC, nabixmols, and nabilone have evidence for targeting global cannabis withdrawal. Nabilone may be useful in preventing relapse but has yet to be tested in the clinical setting, and nabixmols requires further study with maintenance dosing in the outpatient setting to determine its longitudinal effects on cannabis use. Topiramate (though poorly tolerated in adolescents), gabapentin, NAC, and chronic dosing of naltrexone have demonstrated reductions in CUD or prevention of relapse in small (eg, gabapentin) or targeted patient samples (eg, NAC in adolescents) but have not yet shown efficacy in larger, adult samples. The recent trial of oxytocin, while demonstrating some positive results for enhancing existing evidence-based psychotherapy, is still too early to determine its clinical utility. Figure 1 visually depicts the current medications that have sufficient evidence in the off-label treatment of CUD and cannabis withdrawal.

Looking at the nuances of each of these trials, including key secondary analysis findings and taking into account systems of care issues, such as costs of medications particularly at higher than FDA-approved doses for nonapproved uses, may prove to be the most fruitful in terms of individualizing current off-label clinical applications for CUD treatment. As some examples, studies have found important differential treatment effects based on gender (Cooper and Haney, 2016; McRae-Clark et al, 2015), age (Gray et al, 2017), severity of use (Mason et al, 2012; Bentzley et al, 2016), impulsivity (Bentzley et al, 2016), medication adherence (Bentzley et al, 2016), and tobacco cigarette smoking (Haney et al, 2013a). Accounting for these key factors and identifying additional ones in future research serves three functions: (1) presents an opportunity to target these factors directly with other treatments to improve the effectiveness of a current treatment; (2) match subgroups of patients to treatments that may be more helpful; and/or (3) measure and publish the results of these characteristics in treatment trials for CUD to better our understanding. These factors also suggest that no one treatment will likely be sufficient for all patients. Targeting these patient characteristics that differentially impact treatment will likely improve treatment outcomes.

\section{Commonalities Across Studies}

The studies to-date all share inclusion of severe spectrum cannabis use, high attrition over the course of the study even in the context of psychosocial interventions to improve engagement, and low rates of abstinence. These commonalities present obstacles with regards to the identified goal of finding medications to treat CUD. It should be noted that many of the negative clinical trials for candidate medications in the treatment of CUD were under-powered, due to a small number of participants randomized, in conjunction with high attrition over the course of the study. As a result, these negative trials are not definitive in excluding medications in the treatment of CUD.

\section{Heavy Cannabis Users}

While inclusion of heavy cannabis users who meet criteria for cannabis dependence by DSM-IV (as compared to abuse) or severe CUD by DSM-V potentially increases the likelihood of detecting a difference in reduction in cannabis use in addition to ensuring successful treatment across the severity of illness in the context of a positive trial, it also targets a population that is likely more refractory to treatment and less responsive to interventions. Patients with more severe/'heavy' substance use are generally more difficult to treat (Simoneau and Brochu, 2017). As a consequence, finding a clinical signal is less likely as the patients are less likely to respond to the intervention. These negative trials may be 'excluding' medications based on their negative results in the treatment of severe CUD while they may demonstrate clinical utility and positive results if used in the treatment of mild to moderate CUD. It is possible that some of the medications investigated to date would prove to be more effective in mild CUD and lighter users who are looking to abstain or reduce use.

\section{High Attrition}

Researchers have attributed the high dropout rates to the fluctuating and low motivation to quit or reduce use in addition to ambivalence to remain in treatment (Hughes et al, 2008; Levin et al, 2011). Several investigators have noted that although heavy marijuana users meet criteria for CUD, they are less likely than other substance use disorder populations to endorse the need for treatment (Levin et al, 2011). In addition, given that cannabis users commonly make numerous unsuccessful quit attempts independently (Hughes et al, 2008), this may reduce their confidence. While psychosocial interventions, such as MET, have been included in treatment studies to address this, most of the studies continue to see high dropout rates. Continuing to identify baseline characteristics that may predispose individuals to leaving treatment (impulsivity, low confidence, changes in motivations, co-occurring behavioral health disorders like $\mathrm{ADHD}$, and so on) and mitigating them is a promising avenue of future research.

\section{Abstinence as the Main Outcome}

Finally, while many studies have looked at abstinence as the primary outcome, few participants achieve this. More studies have found reductions in use, although often not more so than in the placebo-treated group. As a clinical end point, reduction in use is likely a reasonable goal in the treatment of CUD, and has been a reasonable outcome in the medication treatment studies of other substance use disorders (Garbutt et al, 1999). However, at present, there is not a consensus around what constitutes clinically meaningful reductions in 
TABLE 3 Summary of Placebo-Controlled Clinical Trials of Medications for CUD Treatment

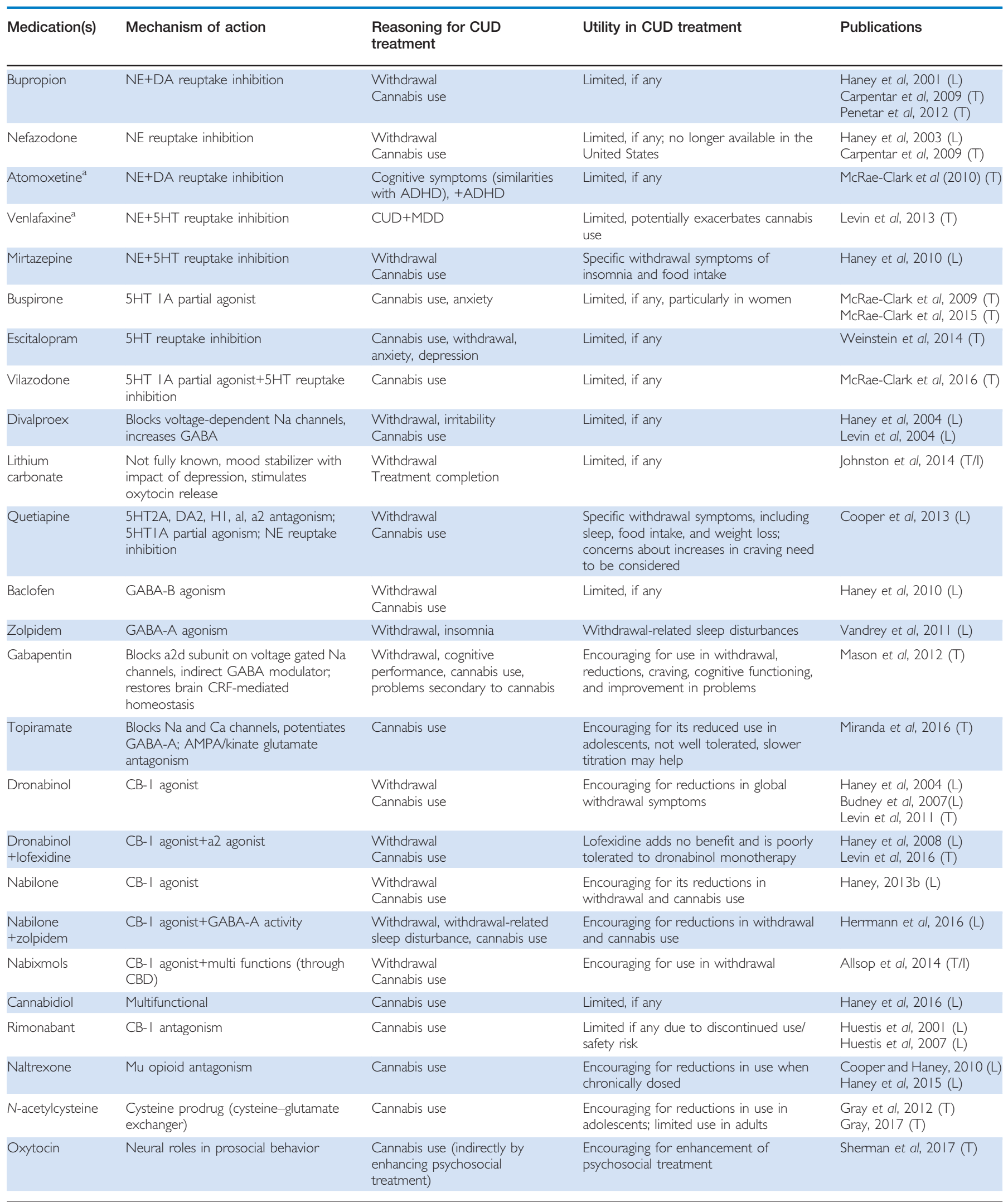

Abbreviations: Ca, calcium; CRF, corticotrophin-releasing factor; DA, dopamine; H, histamine; $5 \mathrm{HT}$, serotonin; I, designates an inpatient treatment study, all others are outpatient; L, identifies human laboratory study; Na, sodium; NE, norepinephrine; T, identifies clinical treatment trial.

aTrials done in CUD+co-occurring disorder. 
Targets and Off-label Medications for Cannabis Use Disorder Treatment

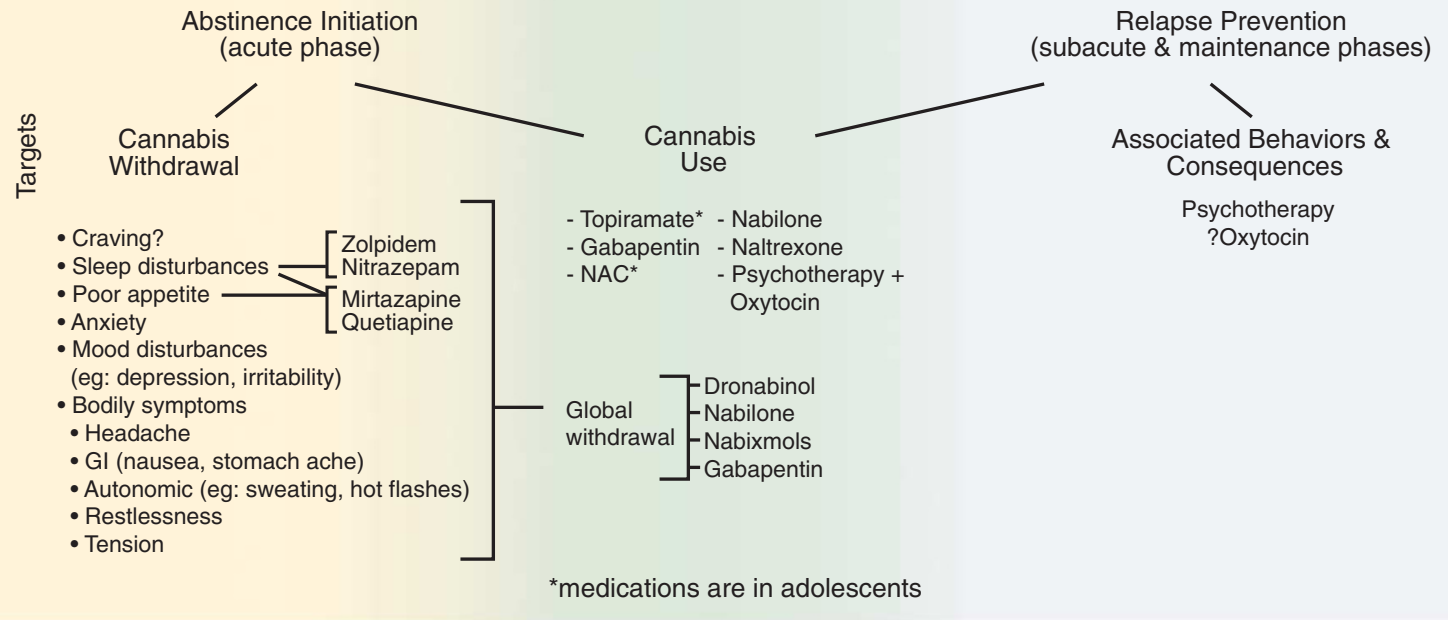

Figure 1. Medications and their targets for off-label use in cannabis use disorder treatment.

cannabis use. Future research should explore functional outcomes that correlate with changes in cannabis use to better inform our conclusions about promising medications for CUD. Ideally, to do this, research is needed to better quantify objective measures of changes in cannabis use through biological assays of bodily fluids (urine, saliva, and blood) and link these changes to patient-centered subjective outcomes, such as functioning or quality of life. While most of the clinical treatment studies measure creatinine-adjusted urine cannabinoid levels and report 'negative' urine testing, none quantify how self-reported reductions in use correlate with changes in the urine cannabinoid levels. Further, human laboratory work has demonstrated that time of day, in addition to other factors, influence urine cannabinoids, which is currently not being addressed in the treatment setting (Smith et al, 2009). Urine cannabinoid levels are the only biological assay currently being assessed in the treatment trials. Future work might consider incorporating other biological assays, such as saliva, where there is great interest given its ease of supervised collection and potential for road-side testing (Lee, 2014). Algorithms of changes in biological cannabinoid levels and self-report may be developed to get a more detailed understanding of changes in cannabis use.

\section{The Research Process}

There also emerge some general issues that should be explored around the process of research to better understand future directions for the field. First, much of the rationale for medication selection has been phenotypically driven in the clinical trials for CUD in top-down process, based on gross observations of symptoms, rather than a bottom-up process, based on the underlying neurobiology. One such example includes the selection of bupropion as a target agent. Observing that nicotine and cannabis withdrawal have overlapping symptoms (phenotypes) led to the hypothesis that a medication for tobacco-smoking cessation may help with cannabis-smoking cessation, but those symptoms are caused by relative or absolute reductions in very different biochemicals. A well-developed and cultivated partnership between the preclinical models of CUD medication treatment with the clinical research groups will allow for a more cohesive and systematic approach to medications development for CUD. Until this occurs, we will likely continue to be dependent on using clinical similarities as a crude compass. Following this, the rationale behind the selection of candidate medications is not always rigorously connected in a clear way to the outcome measures used or the study design. If a medication is postulated to be effective because it may improve symptoms of cannabis withdrawal that would thereby reduce use, cannabis withdrawal measure(s) should be tracked in conjunction with changes in cannabis use. A challenge we face (at least for now) is our dependency on clinical presentation and self-report of symptoms (phenotypes) that overlap with fluctuations in human behavior, experiences, and co-occurring disorders, particularly outside the human lab and in the clinical treatment setting. Given this, our scientific approach needs to be consistent with our reasoning and attempt to disentangle the many possible factors contributing to observed behaviors and self-report of symptoms. To accomplish this, we as a field, should agree upon measures to use across studies and how to report them, particularly as it relates to withdrawal symptoms if withdrawal is a consideration in the mechanism of action by which the medication has an effect. To this end, as society changes and policies shape the current cannabis landscape, how individuals consume cannabis evolves. The access to edibles, vaping, and dabbing all have the potential to change pharmacokinetics and pharmacodynamics of THC in addition to the impact they may have on overall volume of cannabis consumed. Quantifying this both biologically and 
through self-report needs to be considered in study design. The best metrics for reporting cannabis use should be determined to reach a consensus in the field, as there are currently a range of ways in which this is reported (eg, 2 consecutive weeks of abstinence, percentage of negative urines, so on). As an example while most studies consider grams of cannabis used per day, consideration into other metrics, such as dollars per day, may be more accurate assuming higher-potency THC products are more expensive. At present, the most reliable approach may be length of use each day or frequency of days of use per week.

Second, increased coordination of the work conducted in the laboratory and the clinical treatment settings may increase the positive (and negative) predictive value of the laboratory findings. If the human laboratory setting is to serve as the first line in predicting the safety and possible efficacy of a medication in treating CUD, a model that includes abstinence initiation (as well as relapse prevention) and a dosing schedule that is more likely to be adhered to (one or at most twice a day dosing) may be more likely to demonstrate clinical benefit from a purported therapeutic medication in the outpatient treatment environment. While many patients might benefit from an inpatient admission for the treatment of CUD, the reality is that at least in the United States, $86 \%$ of patients presenting with cannabis as the primary problem are referred for treatment in an ambulatory care setting, with either reduction in use or abstinence initiation as the first step in treatment (SAMHSA, 2016). Thus, targeting relapse prevention without including abstinence initiation in the laboratory setting may not have direct applicability to many of the CUD patients seeking treatment. Further, as CUD is a chronic disorder, medication treatment, even in the acute and subacute phases of clinical treatment, will be dosed more than once. As seen in the lab studies of acute $v s$ chronic dosing of naltrexone, laboratory medication dosing should also, as much as possible, closely mirror treatment in real-world settings. In addition, while participant populations across laboratory and clinical trials share similarities with regards to the extent of their cannabis use, criteria for CUD, and related consequences, there are a number of important differences identified between treatment-seeking and non-treatment-seeking cannabis users that may impact the effects of medication (Pacek and Vandrey, 2014). As identified in current studies, differences in gender, age, history and frequency of use, quit attempts and duration, experience of withdrawal symptoms, and number of reasons for making quit attempts are different in participants in the human laboratory as compared to the treatment research setting (Pacek and Vandrey, 2014). The overall monetary compensation (a strong driver of human behavior) differs across human laboratory and clinical treatment studies as well. Future studies might consider modeling abstinence initiation or reduction in use in the laboratory setting, consistently using chronic medication dosing, and brainstorming thoughtful and ethically designed hybrid laboratory-treatment studies.

\section{More than Withdrawal}

Finally, it appears that treating withdrawal is not sufficient as a monotherapy to reduce use and initiate abstinence, at least in severe cannabis users. Future work should make use of medications with different proposed mechanisms. Some candidate medications include fatty acid amide hydrolase inhibitors, serotonin $2 \mathrm{c}$ agonists, and high-affinity CB-1 partial agonists. As with most chronic diseases such as diabetes, hypertension, or major depressive disorder, only the mild spectrum of pathology is easily treated with monotherapy, while more moderate to severe cases, such as those currently representative in CUD clinical trials, require combination strategies that work by different mechanisms of action to generate improvements. The combination results in synergies that ultimately have a clinically meaningful effect. While some studies have begun to explore medication combinations, more work is needed in this area.

\section{FUNDING AND DISCLOSURE}

The authors declare no conflict of interest.

\section{ACKNOWLEDGMENTS}

Dr Brezing is supported by T32DA007294. Dr Levin received medication from US WorldMed for past research trials, served as a consultant to GW Pharmaceuticals (2012-2013) and Shire/Eli Lily (2005-2007). Dr. Levin's work is supported by the following grants: K24 DA 029647 and T32 DA 007294 from NIDA.

\section{REFERENCES}

Addolorato G, Caputo F, Capristo E, Colombo G, Gessa GL, Gasbarrini G (2000). Ability of baclofen in reducing alcohol craving and intake: I-preliminary clinical evidence. Alcohol Clin Exp Res 24: 67-71.

Allsop DJ, Copeland J, Norberg MM, Fu S, Molnar A, Lewis J et al (2012). Quantifying the clinical significance of cannabis withdrawal. PLOS ONE 7: e44864.

Allsop DJ, Norberg MM, Copeland J, Fu S, Budney AJ (2011). The Cannabis Withdrawal Scale development: patterns and predictors of cannabis withdrawal and distress. Drug Alcohol Depend 119: 123-129.

Allsop DJ, Bartlett DJ, Johnston J, Helliwell D, Winstock A, McGregor IS et al (2015). The effects of lithium carbonate supplemented with nitrazepam on sleep disturbance during cannabis abstinence. J Clin Sleep Med 11: 1153-1162.

Allsop DJ, Copeland J, Lintzeris N, Dunlop AJ, Montebello M, Sadler C et al (2014). Nabiximols as an agonist replacement therapy during cannabis withdrawal: a randomized clinical trial. JAMA Psychiatry 71: 281-291. This study demonstrated the utility of nabixmols in the inpatient treatment of cannabis withdrawal.

Anthenelli RM, Heffner JL, Wong E, Tibbs J, Russell K, Isgro M et al (2017). A randomized trial evaluating whether topiramate aids smoking cessation and prevents alcohol relapse in recovering alcohol-dependent men. Alcohol Clin Exp Res 41: 197-206.

APA (2013). Diagnostic and Statistical Manual of Mental Disorders. American Psychiatric Association: Arlington, VA, USA.

Balter RE, Cooper ZD, Haney M (2014). Novel pharmacologic approaches to treating cannabis use disorder. Curr Addict Rep 1: 137-143.

Bedi G, Cooper ZD, Haney M (2013). Subjective, cognitive and cardiovascular doseeffect profile of nabilone and dronabinol in marijuana smokers. Addict Biol 18: 872-881.

Benn A, Robinson ES (2017). Differential roles for cortical versus sub-cortical noradrenaline and modulation of impulsivity in the rat. Psychopharmacology 234: 255-266.

Bentzley JP, Tomko RL, Gray KM (2016). Low pretreatment impulsivity and high medication adherence increase the odds of abstinence in a trial of 
$\mathrm{N}$-acetylcysteine in adolescents with cannabis use disorder. J Subst Abuse Treat 63: 72-77. This secondary analysis identified factors that may ultimately influence the results of clinical trials and guide future research.

Bonnet U, Preuss UW (2017). The cannabis withdrawal syndrome: current insights. Subst Abuse Rehabil 8: 9-37.

Buckner JD, Carroll KM (2010). Effect of anxiety on treatment presentation and outcome: results from the Marijuana Treatment Project. Psychiatry Res 178: 493-500.

Buckner JD, Schmidt NB, Lang AR, Small JW, Schlauch RC, Lewinsohn PM (2008). Specificity of social anxiety disorder as a risk factor for alcohol and cannabis dependence. J Psychiatric Res 42: 230-239.

Budney AJ, Novy PL, Hughes JR (1999). Marijuana withdrawal among adults seeking treatment for marijuana dependence. Addiction 94: 1311-1322.

Budney AJ, Higgins ST, Radonovich KJ, Novy PL (2000). Adding voucher-based incentives to coping skills and motivational enhancement improves outcomes during treatment for marijuana dependence. J Consult Clin Psychol 68: 1051-1061.

Budney AJ, Moore BA, Rocha HL, Higgins ST (2006). Clinical trial of abstinencebased vouchers and cognitive-behavioral therapy for cannabis dependence. $J$ Consult Clin Psychol 74: 307-316.

Budney AJ, Vandrey RG, Hughes JR, Moore BA, Bahrenburg B (2007). Oral delta-9tetrahydrocannabinol suppresses cannabis withdrawal symptoms. Drug Alcohol Depend 86: 22-29.

Budney AJ, Vandrey RG, Hughes JR, Thostenson JD, Bursac Z (2008). Comparison of cannabis and tobacco withdrawal: severity and contribution to relapse. J Subst Abuse Treat 35: 362-368.

Carpenter KM, McDowell D, Brooks DJ, Cheng WY, Levin FR (2009). A preliminary trial: double-blind comparison of nefazodone, bupropion-SR, and placebo in the treatment of cannabis dependence. Am J Addict 18: 53-64.

Cooper ZD, Foltin RW, Hart CL, Vosburg SK, Comer SD, Haney M (2013). A human laboratory study investigating the effects of quetiapine on marijuana withdrawal and relapse in daily marijuana smokers. Addict Biol 18: 993-1002.

Cooper ZD, Haney M (2010). Opioid antagonism enhances marijuana's effects in heavy marijuana smokers. Psychopharmacology 211: 141-148.

Cooper ZD, Haney M (2016). Sex-dependent effects of cannabis-induced analgesia. Drug Alcohol Depend 167: 112-120.

Copeland J, Swift W, Roffman R, Stephens R (2001). A randomized controlled trial of brief cognitive-behavioral interventions for cannabis use disorder. J Subst Abuse Treat 21: 55-64 discussion 65-56.

Cornelius JR, Bukstein OG, Douaihy AB, Clark DB, Chung TA, Daley DC et al (2010). Double-blind fluoxetine trial in comorbid MDD-CUD youth and young adults. Drug Alcohol Depend 112: 39-45.

Cui SS, Bowen RC, Gu GB, Hannesson DK, Yu PH, Zhang X (2001). Prevention of cannabinoid withdrawal syndrome by lithium: involvement of oxytocinergic neuronal activation. J Neurosci 21: 9867-9876.

Degenhardt L, Ferrari AJ, Calabria B, Hall WD, Norman RE, McGrath J et al (2013). The global epidemiology and contribution of cannabis use and dependence to the global burden of disease: results from the GBD 2010 study. PLOS ONE 8: e76635.

Dennis M, Babor TF, Roebuck MC, Donaldson J (2002). Changing the focus: the case for recognizing and treating cannabis use disorders. Addiction 97(Suppl 1): 4-15.

Garbutt JC, West SL, Carey TS, Lohr KN, Crews FT (1999). Pharmacological treatment of alcohol dependence: a review of the evidence. JAMA 281: 1318-1325.

Gates PJ, Sabioni P, Copeland J, Le Foll B, Gowing L (2016). Psychosocial interventions for cannabis use disorder. Cochrane Database Syst Rev CD005336.

Ghaemi SN, Katzow JJ, Desai SP, Goodwin FK (1998). Gabapentin treatment of mood disorders: a preliminary study. J Clin Psychiatry 59: 426-429.

Gomes FV, Resstel LB, Guimaraes FS (2011). The anxiolytic-like effects of cannabidiol injected into the bed nucleus of the stria terminalis are mediated by 5-HT1A receptors. Psychopharmacology 213: 465-473.

Gorelick DA (2016). Pharmacological treatment of cannabis-related disorders: a narrative review. Curr Pharm Des 22: 6409-6419.

Gray KM, Carpenter MJ, Baker NL, DeSantis SM, Kryway E, Hartwell KJ et al (2012). A double-blind randomized controlled trial of $\mathrm{N}$-acetylcysteine in cannabisdependent adolescents. Am J Psychiatry 169: 805-812. This is the first positive fully powered placebo controlled trial of a medication for CUD.

Gray KM, Sonne SC, McClure EA, Ghitza UE, Matthews AG, McRae-Clark AL et al (2017). A randomized placebo-controlled trial of $\mathrm{N}$-acetylcysteine for cannabis use disorder in adults. Drug Alcohol Depend 177: 249-257.

Gray KM, Watson NL, Carpenter MJ, Larowe SD (2010). N-acetylcysteine (NAC) in young marijuana users: an open-label pilot study. Am J Addict 19: 187-189.

Gueye AB, Pryslawsky Y, Trigo JM, Poulia N, Delis F, Antoniou K et al (2016). The CB1 neutral antagonist AM4113 retains the therapeutic efficacy of the inverse agonist rimonabant for nicotine dependence and weight loss with better psychiatric tolerability. Int J Neuropsychopharmacol 19: 1-11.
Haney M, Hart CL, Foltin RW (2006). Effects of baclofen on cocaine selfadministration: opioid- and nonopioid-dependent volunteers. Neuropsychopharmacology 31: 1814-1821.

Haney M, Bedi G, Cooper ZD, Glass A, Vosburg SK, Comer SD et al (2013a). Predictors of marijuana relapse in the human laboratory: robust impact of tobacco cigarette smoking status. Biol Psychiatry 73: 242-248.

Haney M, Cooper ZD, Bedi G, Vosburg SK, Comer SD, Foltin RW (2013b). Nabilone decreases marijuana withdrawal and a laboratory measure of marijuana relapse. Neuropsychopharmacology 38: 1557-1565. This human laboratory study identified nabilone as a promising agent for future treatment trial research in the treatment of CUD.

Haney M, Hart CL, Vosburg SK, Comer SD, Reed SC, Cooper ZD et al (2010). Effects of baclofen and mirtazapine on a laboratory model of marijuana withdrawal and relapse. Psychopharmacology 211: 233-244.

Haney M, Hart CL, Vosburg SK, Comer SD, Reed SC, Foltin RW (2008). Effects of $\mathrm{THC}$ and lofexidine in a human laboratory model of marijuana withdrawal and relapse. Psychopharmacology 197: 157-168.

Haney M, Hart CL, Vosburg SK, Nasser J, Bennett A, Zubaran C et al (2004). Marijuana withdrawal in humans: effects of oral THC or divalproex. Neuropsychopharmacology 29: 158-170.

Haney M, Hart CL, Ward AS, Foltin RW (2003). Nefazodone decreases anxiety during marijuana withdrawal in humans. Psychopharmacology 165: 157-165.

Haney M, Malcolm RJ, Babalonis S, Nuzzo PA, Cooper ZD, Bedi G et al (2016). Oral cannabidiol does not alter the subjective, reinforcing or cardiovascular effects of smoked cannabis. Neuropsychopharmacology 41: 1974-1982.

Haney M, Ramesh D, Glass A, Pavlicova M, Bedi G, Cooper ZD (2015). Naltrexone maintenance decreases cannabis self-administration and subjective effects in daily cannabis smokers. Neuropsychopharmacology 40: 2489-2498. This human laboratory study of naltrexone demonstrated the importance of chronic dosing and its effects on outcomes.

Haney M, Spealman R (2008). Controversies in translational research: drug selfadministration. Psychopharmacology 199: 403-419.

Haney M, Ward AS, Comer SD, Hart CL, Foltin RW, Fischman MW (2001). Bupropion SR worsens mood during marijuana withdrawal in humans. Psychopharmacology 155: 171-179.

Hart CL, Haney M, Vosburg SK, Comer SD, Foltin RW (2005). Reinforcing effects of oral Delta9-THC in male marijuana smokers in a laboratory choice procedure. Psychopharmacology 181: 237-243.

Hart CL, Ward AS, Haney M, Comer SD, Foltin RW, Fischman MW (2002). Comparison of smoked marijuana and oral Delta(9)-tetrahydrocannabino in humans. Psychopharmacology 164: 407-415.

Herrmann ES, Cooper ZD, Bedi G, Ramesh D, Reed SC, Comer SD et al (2016). Effects of zolpidem alone and in combination with nabilone on cannabis withdrawal and a laboratory model of relapse in cannabis users. Psychopharmacology 233: 2469-2478.

Hill MN, Sun JC, Tse MT, Gorzalka BB (2006). Altered responsiveness of serotonin receptor subtypes following long-term cannabinoid treatment. Int J Neuropsychopharmacol 9: 277-286.

Huestis MA, Boyd SJ, Heishman SJ, Preston KL, Bonnet D, Le Fur G et al (2007). Single and multiple doses of rimonabant antagonize acute effects of smoked cannabis in male cannabis users. Psychopharmacology 194: 505-515.

Huestis MA, Gorelick DA, Heishman SJ, Preston KL, Nelson RA, Moolchan ET et al (2001). Blockade of effects of smoked marijuana by the CB1-selective cannabinoid receptor antagonist SR141716. Arch Gen Psychiatry 58: 322-328.

Hughes JR, Naud S, Budney AJ, Fingar JR, Callas PW (2016). Attempts to stop or reduce daily cannabis use: an intensive natural history study. Psychol Addict Behav 30: 389-397.

Hughes JR, Peters EN, Callas PW, Budney AJ, Livingston AE (2008). Attempts to stop or reduce marijuana use in non-treatment seekers. Drug Alcohol Depend 97 180-184.

Johnston J, Lintzeris N, Allsop DJ, Suraev A, Booth J, Carson DS et al (2014). Lithium carbonate in the management of cannabis withdrawal: a randomized placebocontrolled trial in an inpatient setting. Psychopharmacology 231: 4623-4636.

Kampman KM, Rukstalis M, Ehrman R, McGinnis DE, Gariti P, Volpicelli JR et al (1999). Open trials as a method of prioritizing medications for inclusion in controlled trials for cocaine dependence. Addict Behav 24: 287-291.

Kelly MA, Pavlicova M, Glass A, Mariani JJ, Bisaga A, Sullivan MA et al (2014). Do withdrawal-like symptoms mediate increased marijuana smoking in individuals treated with venlafaxine-XR? Drug Alcohol Depend 144: 42-46.

Keuroghlian AS, Barry AS, Weiss RD (2012). Circadian dysregulation, zolpidem dependence, and withdrawal seizure in a resident physician performing shift work. Am J Addict 21: 576-577.

Lee D, Vandrey R, Mendu DR, Murray JA, Barnes AJ, Huestis MA (2015). Oral fluid cannabinoids in chronic frequent cannabis smokers during ad libitum cannabis smoking. Drug Test Anal 7: 494-501. 
Levin FR, Mariani J, Brooks DJ, Pavlicova M, Nunes EV, Agosti $V$ et al (2013). A randomized double-blind, placebo-controlled trial of venlafaxine-extended release for co-occurring cannabis dependence and depressive disorders. Addiction 108: 1084-1094.

Levin FR, Mariani JJ, Brooks DJ, Pavlicova M, Cheng W, Nunes EV (2011). Dronabinol for the treatment of cannabis dependence: a randomized, doubleblind, placebo-controlled trial. Drug Alcohol Depend 116: 142-150. This fully powered clinical trial, though negative, demonstrated the positive predictive validity of the human laboratory studies.

Levin FR, Mariani JJ, Pavlicova M, Brooks D, Glass A, Mahony A et al (2016). Dronabinol and lofexidine for cannabis use disorder: a randomized, double-blind, placebo-controlled trial. Drug Alcohol Depend 159: 53-60. Guided by the human laboratory, this study highlights the importance of considering practical constraints in the clinical treatment setting that may not be evident or fully accounted for in the lab setting.

Levin FR, McDowell D, Evans SM, Nunes E, Akerele E, Donovan S et al (2004). Pharmacotherapy for marijuana dependence: a double-blind, placebo-controlled pilot study of divalproex sodium. Am J Addict 13: 21-32. Though negative, this was the first clinical treatment trial for CUD.

Lo HS, Yang CM, Lo HG, Lee CY, Ting H, Tzang BS (2010). Treatment effects of gabapentin for primary insomnia. Clin Neuropharmacol 33: 84-90.

Mason BJ, Light JM, Williams LD, Drobes DJ (2009). Proof-of-concept human laboratory study for protracted abstinence in alcohol dependence: effects of gabapentin. Addict Biol 14: 73-83.

Mariani JJ, Pavlicova M, Mamczur AK, Bisaga A, Nunes EV, Levin FR (2014). Openlabel pilot study of quetiapine treatment for cannabis dependence. Am J Drug Alcohol Abuse 14: 280-284.

Martino S, Ball SA, Nich C, Frankforter TL, Carroll KM (2009). Informal discussions in substance abuse treatment sessions. J Subst Abuse Treat 36: 366-375.

Mason BJ, Crean R, Goodell V, Light JM, Quello S, Shadan F et al (2012). A proofof-concept randomized controlled study of gabapentin: effects on cannabis use, withdrawal and executive function deficits in cannabis-dependent adults. Neuropsychopharmacology 37: 1689-1698. This pilot placebo controlled trial demonstrated the promise of gabapentin in the treatment of CUD by targeting withdrawal, executive functioning, and reductions in use.

McRae-Clark AL, Baker NL, Gray KM, Killeen T, Hartwell KJ, Simonian SJ (2016). Vilazodone for cannabis dependence: a randomized, controlled pilot trial. Am J Addict 25: 69-75.

McRae-Clark AL, Baker NL, Gray KM, Killeen TK, Wagner AM, Brady KT et al (2015). Buspirone treatment of cannabis dependence: a randomized, placebocontrolled trial. Drug Alcohol Depend 156: 29-37.

McRae-Clark AL, Baker NL, Maria MM, Brady KT (2013). Effect of oxytocin on craving and stress response in marijuana-dependent individuals: a pilot study. Psychopharmacology 228: 623-631.

McRae-Clark AL, Carter RE, Killeen TK, Carpenter MJ, Wahlquist AE, Simpson SA et al (2009). A placebo-controlled trial of buspirone for the treatment of marijuana dependence. Drug Alcohol Depend 105: 132-138.

McRae-Clark AL, Carter RE, Killeen TK, Carpenter MJ, White KG, Brady KT (2010). A placebo-controlled trial of atomoxetine in marijuana-dependent individuals with attention deficit hyperactivity disorder. Am J Addict 19: 481-489.

Mills EJ, Wu P, Lockhart I, Thorlund K, Puhan M, Ebbert JO (2012). Comparisons of high-dose and combination nicotine replacement therapy, varenicline, and bupropion for smoking cessation: a systematic review and multiple treatment meta-analysis. Ann Med 44: 588-597.

Miranda R Jr, Treloar H, Blanchard A, Justus A, Monti PM, Chun T et al (2016). Topiramate and motivational enhancement therapy for cannabis use among youth: a randomized placebo-controlled pilot study. Addict Biol 22: 779-790. Though poorly tolerated in adolescents, this pilot placebocontrolled study did show that topiramate may have a role in reducing cannabis use.

Morales P, Reggio PH, Jagerovic N (2017). An overview on medicinal chemistry of synthetic and natural derivatives of cannabidiol. Front Pharmacol 8: 422.

Moss HB, Chen CM, Yi HY (2012). Measures of substance consumption among substance users, DSM-IV abusers, and those with DSM-IV dependence disorders in a nationally representative sample. J Stud Alcohol Drugs 73: 820-828.

Olmstead TA, Abraham AJ, Martino S, Roman PM (2012). Counselor training in several evidence-based psychosocial addiction treatments in private US substance abuse treatment centers. Drug Alcohol Depend 120: 149-154.

Pacek LR, Vandrey R (2014). Cannabis use history and characteristics of quit attempts: a comparison study of treatment-seeking and non-treatment-seeking cannabis users. Exp Clin Psychopharmacol 22: 517-523.
Penetar DM, Looby AR, Ryan ET, Maywalt MA, Lukas SE (2012). Bupropion reduces some of the symptoms of marihuana withdrawal in chronic marihuana users: a pilot study. Subst Abuse 6: 63-71.

Perkonigg A, Goodwin RD, Fiedler A, Behrendt S, Beesdo K, Lieb R et al (2008). The natural course of cannabis use, abuse and dependence during the first decades of life. Addiction 103: 439-449 discussion 450-451.

Roberfroid D, Lachat C, Lucet C (2010). Termination of the CRESCENDO trial. Lancet 376: 1983-1984 author reply 1984-1985.

Roberto M, Gilpin NW, O'Dell LE, Cruz MT, Morse AC, Siggins GR et al (2008). Cellular and behavioral interactions of gabapentin with alcohol dependence. J Neurosci 28: 5762-5771.

SAMHSA (2015). Behavioral Health Trends in the United States: Results from the 2014 National Survey on Drug Use and Health NSDUH Series H-50 Center for Behavioral Health Statistics and Quality: Rockville, MD, USA.

SAMHSA (2016). Treatment Episode Data Set (TEDS): 2004-2014. Substance Abuse and Mental Health Services Administration, Center for Behavioral Health Statistics and Quality: Rockville, MD, USA

Santa Ana EJ, Martino S, Ball SA, Nich C, Frankforter TL, Carroll KM (2008). What is usual about "treatment-as-usual"? Data from two multisite effectiveness trials. J Subst Abuse Treat 35: 369-379.

Sherman BJ, Baker NL, McRae-Clark AL (2017). Effect of oxytocin pretreatment on cannabis outcomes in a brief motivational intervention. Psychiatry Res 249: 318-320.

Sherman BJ, McRae-Clark AL (2016). Treatment of cannabis use disorder: current science and future outlook. Pharmacotherapy 36: 511-535.

Simoneau H, Brochu S (2017). ASI profile of persons who re-enter treatment for substance use disorders. Subst Abuse 1-6.

Smith ML, Barnes AJ, Huestis MA (2009). Identifying new cannabis use with urine creatinine-normalized $\mathrm{THCCOOH}$ concentrations and time intervals between specimen collections. J Anal Toxicol 33: 185-189.

Stead LF, Perera R, Bullen C, Mant D, Lancaster T (2008). Nicotine replacement therapy for smoking cessation. Cochrane Database Syst Rev CD000146.

Tait RJ, Spijkerman R, Riper H (2013). Internet and computer based interventions for cannabis use: a meta-analysis. Drug Alcohol Depend 133: 295-304.

Tirado CF, Goldman M, Lynch K, Kampman KM, Obrien CP (2008). Atomoxetine for treatment of marijuana dependence: a report on the efficacy and high incidence of gastrointestinal adverse events in a pilot study. Drug Alcohol Depend 94: 254-257.

Trigo JM, Lagzdins D, Rehm J, Selby P, Gamaleddin I, Fischer B et al (2016a). Effects of fixed or self-titrated dosages of Sativex on cannabis withdrawal and cravings. Drug Alcohol Depend 161: 298-306.

Trigo JM, Soliman A, Staios G, Quilty L, Fischer B, George TP et al (2016b). Sativex associated with behavioral-relapse prevention strategy as treatment for cannabis dependence: a case series. J Addict Med 10: 274-279.

United Nations Office on Drugs and Crime (2015). World Drug Report 2015. United Nations Publication: Vienna, Austria.

Vandrey R, Haney M (2009). Pharmacotherapy for cannabis dependence: how close are we? CNS Drugs 23: 543-553.

Vandrey R, Smith MT, McCann UD, Budney AJ, Curran EM (2011). Sleep disturbance and the effects of extended-release zolpidem during cannabis withdrawal. Drug Alcohol Depend 117: 38-44.

Vandrey R, Stitzer ML, Mintzer MZ, Huestis MA, Murray JA, Lee D (2013). The dose effects of short-term dronabinol (oral THC) maintenance in daily cannabis users. Drug Alcohol Depend 128: 64-70.

Vandrey RG, Budney AJ, Hughes JR, Liguori A (2008). A within-subject comparison of withdrawal symptoms during abstinence from cannabis, tobacco, and both substances. Drug Alcohol Depend 92: 48-54.

Vermersch P (2011). Sativex((R)) (tetrahydrocannabinol+cannabidiol), an endocannabinoid system modulator: basic features and main clinical data. Expert Rev Neurother 11: 15-19.

Wei D, Lee D, Cox CD, Karsten CA, Penagarikano O, Geschwind DH et al (2015). Endocannabinoid signaling mediates oxytocin-driven social reward. Proc Natl Acad Sci USA 112: 14084-14089.

Weinstein AM, Miller H, Bluvstein I, Rapoport E, Schreiber S, Bar-Hamburger R et al (2014). Treatment of cannabis dependence using escitalopram in combination with cognitive-behavior therapy: a double-blind placebo-controlled study. Am J Drug Alcohol Abuse 40: 16-22.

Winstock AR, Lea T, Copeland J (2009). Lithium carbonate in the management of cannabis withdrawal in humans: an open-label study. J Psychopharmacol 23: 84-93.

Zanelati TV, Biojone C, Moreira FA, Guimaraes FS, Joca SR (2010). Antidepressantlike effects of cannabidiol in mice: possible involvement of $5-\mathrm{HT} 1 \mathrm{~A}$ receptors. $\mathrm{Br} \mathrm{J}$ Pharmacol 159: 122-128. 